University of Nebraska - Lincoln

DigitalCommons@University of Nebraska - Lincoln

$10-2007$

\title{
Wigner Distribution of a Transducer Beam Pattern Within a Multiple Scattering Formalism for Heterogeneous Solids
}

\author{
Goutam Ghoshal \\ University of Nebraska - Lincoln \\ Joseph A. Turner \\ University of Nebraska - Lincoln, jaturner@unl.edu \\ Richard Weaver \\ University of Illinois at Urbana-Champaign, 1110 W Green Street, Urbana, Illinois
}

Follow this and additional works at: https://digitalcommons.unl.edu/engineeringmechanicsfacpub

Part of the Mechanical Engineering Commons

\begin{abstract}
Ghoshal, Goutam; Turner, Joseph A.; and Weaver, Richard, "Wigner Distribution of a Transducer Beam Pattern Within a Multiple Scattering Formalism for Heterogeneous Solids" (2007). Faculty Publications from the Department of Engineering Mechanics. 41.

https://digitalcommons.unl.edu/engineeringmechanicsfacpub/41
\end{abstract}

This Article is brought to you for free and open access by the Mechanical \& Materials Engineering, Department of at DigitalCommons@University of Nebraska - Lincoln. It has been accepted for inclusion in Faculty Publications from the Department of Engineering Mechanics by an authorized administrator of DigitalCommons@University of Nebraska - Lincoln. 


\title{
Wigner distribution of a transducer beam pattern within a multiple scattering formalism for heterogeneous solids
}

\author{
Goutam Ghoshal and Joseph A. Turner ${ }^{a)}$ \\ Department of Engineering Mechanics, W317.4 Nebraska Hall, University of Nebraska-Lincoln, \\ Lincoln, Nebraska 68588-0526 \\ Richard L. Weaver \\ Department of Physics, University of Illinois at Urbana-Champaign, $1110 \mathrm{~W}$ Green Street, \\ Urbana, Illinois 61801
}

(Received 11 January 2007; revised 29 July 2007; accepted 30 July 2007)

\begin{abstract}
Diffuse ultrasonic backscatter measurements have been especially useful for extracting microstructural information and for detecting flaws in materials. Accurate interpretation of experimental data requires robust scattering models. Quantitative ultrasonic scattering models include components of transducer beam patterns as well as microstructural scattering information. Here, the Wigner distribution is used in conjunction with the stochastic wave equation to model this scattering problem. The Wigner distribution represents a distribution in space and time of spectral energy density as a function of wave vector and frequency. The scattered response is derived within the context of the Wigner distribution of the beam pattern of a Gaussian transducer. The source and receiver distributions are included in the analysis in a rigorous fashion. The resulting scattered response is then simplified in the single-scattering limit typical of many diffuse backscatter experiments. Such experiments, usually done using a modified pulse-echo technique, utilize the variance of the signals in space as the primary measure of microstructure. The derivation presented forms a rigorous foundation for the multiple scattering process associated with ultrasonic experiments in heterogeneous media. These results are anticipated to be relevant to ultrasonic nondestructive evaluation of polycrystalline and other heterogeneous solids.
\end{abstract}

(c) 2007 Acoustical Society of America. [DOI: 10.1121/1.2773989]

PACS number(s): 43.35.Zc, 43.20.Bi, 43.20.Gp, 43.35.Cg [TDM] Pages: 2009-2021

\section{INTRODUCTION}

Acoustic propagation in random media at wavelengths comparable to or greater than the size of typical heterogeneities is widely studied, often with a view toward characterization of the microstructure. At low frequencies, such that the wavelengths are very long compared with the length scales of the heterogeneity, the medium is an effectively homogeneous continuum. Measurements of acoustic wave speeds, attenuations, and allowed acoustic polarizations can provide a means for material characterization, ${ }^{1-7}$ though microstructural features are not resolved. In such media the chief source of coherent wave attenuation is dissipative and generally unrelated to the microstructures of interest. At moderate frequencies, attenuation is augmented by diffuse scattering ${ }^{8-10}$ out of an acoustic beam leading to the possibility of microstructure characterization by means of the frequency dependence of acoustic velocities and attenuation. At slightly higher frequencies where one expects to find significant dependence on microstructure, there is much less literature. This is largely traceable to the very high mean field attenuations characteristic of this frequency range; coherent propagation is too weak for measurement except in special circumstances. ${ }^{11}$

\footnotetext{
a) Author to whom correspondence should be addressed; electronic mail: jaturner@unl.edu
}

Diffuse multiply scattered fields, however, are not adversely impacted by strong attenuation of the coherent propagation. After many random scatterings or reflections, an acoustic field has lost its original phase coherence, and the field variables become random, with zero mean. The mean square of the field, related to the mean wave energy density, however, remains a meaningful quantity. Diffuse field studies have successfully characterized sources, fields, and media by means of measurements of the evolving wave energy. ${ }^{12-17}$ Ultrasonic diffuse field studies have shown themselves capable of characterization of internal friction in polycrystalline microstructure and associated grain noise, ${ }^{13-17}$ revealing the Anderson localization of the modes of a disordered structure, ${ }^{18}$ and characterization of ultrasonic sources by means of the spectral distribution of the resulting diffuse wave energy. ${ }^{19,20}$

Of particular interest here are the ultrasonic studies of diffuse waves in multiply scattering elastic media. They may be classified in two groups. One concerns work, theoretical ${ }^{21}$ and experimental, ${ }^{17}$ in which the received signal is modeled as consisting entirely of singly scattered contributions. The other assumes, and/or models, the received signal as thoroughly multiply scattered, i.e., as a fully developed diffuse field. ${ }^{16,22-26}$ The former case presumably applies at times sufficiently soon after a transient source has acted, while the latter case applies at times sufficiently long after that source has acted. A clear distinction between the two regimes obvi- 
ously requires that studies be carried out in the time domain. But it also demands that we understand the transition between the regimes, a topic excluded from previous investigations. The first of these demands regarding the time domain analysis has not been met. The second, regarding the transition regimes, is an inconvenience at best, and at worst (if signals at early times are contaminated by electronic cross talk or double scattering, and if late times are inaccessible because of strong absorption) will prohibit application of these diffuse energy methods entirely. For this reason a theory for the transition regime is needed.

Several years ago, it was recognized that diffuse ultrasonic energy, at all times from the singly scattered regime to the fully developed regime, ought to be governed by equations like those already known for the transport of diffuse optical energy through multiply scattering atmospheres ${ }^{27-32}$ and the transport of neutrons through reactors, ${ }^{33-35}$ that is, an equation of radiative transfer. In a series of theoretical papers, Turner and Weaver ${ }^{22,23,21}$ in an ultrasonic context, Papanicolaou and co-workers, ${ }^{36}$ and van Tiggelen and co-workers ${ }^{37,38}$ for seismic waves and elastic waves, ${ }^{39,40} \mathrm{de}$ veloped equations of radiative transfer (RTE) for elastic waves in multiply scattering media and argued by consideration of energy flow and conservation.

The form that these equations take is instructive. The relevant dependent variable is not energy, or even energy flux, but specific intensity. For a scalar wave this quantity has a single component, $I(\mathbf{x}, \hat{\mathbf{n}}, t, \omega)$, which may depend on position $\mathbf{x}$, time $t$, frequency $\omega$, and direction of propagation $\hat{\mathbf{n}}$. $I$ represents the energy flux in a frequency band of unit width, per unit solid angle in direction $\hat{\mathbf{n}}$ at frequency $\omega$ at time $t$ and position $\mathbf{x}$. The scalar RTE for $I$ is given by

$$
\begin{aligned}
& \frac{\partial I(\mathbf{x}, \hat{\mathbf{n}}, t)}{\partial t}+c \hat{\mathbf{n}} \cdot \nabla I(\mathbf{x}, \hat{\mathbf{n}}, t)+\kappa I(\mathbf{x}, \hat{\mathbf{n}}, t) \\
& =\int p(\hat{\mathbf{n}}, \hat{\mathbf{m}}) I(\mathbf{x}, \hat{\mathbf{m}}, t) d^{2} \hat{\mathbf{m}},
\end{aligned}
$$

where the dependence on frequency, $\omega$, has been suppressed.

Equation (1) indicates that $I$ is transported at a speed $c$ in direction $\hat{\mathbf{n}}$, attenuates at a rate (per unit time) $\kappa$, and is augmented by scattering from direction $\hat{\mathbf{m}}$ into direction $\hat{\mathbf{n}}$. The scattering function $p$ is characteristic of the microstructure. The attenuation, $\kappa$, will have contributions from both scattering and dissipation.

For an electromagnetic wave ${ }^{41,27}$ or an elastic wave $e^{22}$ the quantity $I$ has Stokes components, representing the specific intensity in different polarizations. In this case, the scattering function $p$ becomes a Stokes matrix. For elastic waves there are five Stokes components. Equations for elastic wave transport of this form have been derived by Weaver, ${ }^{16}$ by Turner and Weaver, ${ }^{22,23}$ and by Ryzhik et al. ${ }^{36}$

Solution methods, established for applications to optical and neutron transport, are most developed for the planarindependent case corresponding to a steady state planar source of specific intensity. Solutions are obtained with spatial dependence confined to a single dimension. Such limits allow application to a wide class of problems encountered in stellar and planetary atmospheres. In ultrasonics, however, where measurements must be carried out in the time domain, and where insonification is rarely a plane wave, such limits are inapplicable. Numerical solutions of elastic wave RTE have been obtained by Turner and Weaver ${ }^{22,23,21}$ for the steady-state case and for the time domain but have so far been confined to the case of planar insonification. More work is needed.

It is not obvious, however, how an equation like Eq. (1) should be applied to the case of insonification and detection by realistic piezoelectric transducers. It is not difficult to insert, in Eq. (1), a source term corresponding to a pencil beam or a spherical wave in an attempt to model a transducer, but real transducers do not generate intensity in that form. In addition, it is not clear what errors might be induced by so doing. By reciprocity, the same uncertainty applies to the modeling of detection. Furthermore, and perhaps more disturbing, Eq. (1) lends itself with difficulty to a rational interpretation (notwithstanding that it has been derived reasonably). In particular, the dependent variable $I$ is ostensibly defined at arbitrary time and frequency, and at arbitrary position and propagation direction. However, these are complementary independent variables, and any prescription for simultaneous dependence on $t$ and $\omega$, or $\mathbf{x}$ and $\mathbf{n}$ (because propagation direction $\mathbf{n}$ corresponds to the direction of a wave vector $\mathbf{k}$ in spatial Fourier transform space) is suspicious. One might suspect that Eq. (1) involves an approximation akin to those employed in ad hoc signal processing schemes, ${ }^{42}$ which attempt simultaneous decomposition in time and frequency. In any case it is clearly appropriate to revisit the derivation of Eq. (1), but with care taken to insert the transducers themselves into the model from the start. This is the purpose of the present communication.

In Sec. II the problem of mean and mean square signals from model source and receiver distributions in a random medium is formally posed. It is solved by familiar perturbative techniques, involving expansions to leading order in fluctuating material properties. The mean signal (or mean square signal) is shown to be equal to a certain convolution between the mean Green's function (or Green's covariance) and the model transducer functions. A Dyson equation is obtained for the mean Green's function, and a Bethe-Salpeter equation is obtained for the Green's function covariance. The latter equation is expanded in a multiple scattering series and the results inserted into the previously derived convolution expression for the mean square signal. It is found that the mean square signal from the receiver is expressible as a multiple scattering series. Each term of that series involves a concatenation of generalized Wigner distribution functions. ${ }^{43}$ By comparison with the RTE it is found that the mathematics indicates that the potentially irrational concept "specific intensity" is actually a rational concept, a Wigner transform. There are no ad hoc prescriptions for simultaneous time and frequency distributions; the mathematics clearly indicates that it is the Wigner function which plays the role of specific intensity. It furthermore shows that transducers are not to be represented by the specific intensity they generate, but rather the more precise concept, their field's Wigner distribution. 


\section{MATHEMATICAL PRELIMINARIES I: DYSON EQUATION AND THE MEAN GREENS' FUNCTION}

We begin with the governing partial differential equation (PDE) for the Green's function of an elastic medium with constant material density (set to unity) and modulus that varies randomly in space

$$
\left[-\delta_{l i} \frac{\partial^{2}}{\partial t^{2}}+\frac{\partial}{\partial x_{k}} \mu_{k l i j}(\mathbf{x}) \frac{\partial}{\partial x_{j}}\right] G_{i \alpha}(\mathbf{x}, \mathbf{y}, t)=\delta^{3}(\mathbf{x}-\mathbf{y}) \delta(t) \delta_{l \alpha} .
$$

The position-dependent modulus tensor $\mu_{k l i j}(\mathbf{x})$ has a mean value of $C_{k l i j}^{0}$, and fluctuations away from the mean given by $\gamma_{i j k l}(\mathbf{x})=\mu_{i j k l}(\mathbf{x})-C_{i j k l}^{0}$. This form for the PDE corresponds to the case of a random polycrystal, in which case $C^{0}$ is the volume average, or Voigt, effective modulus. If $\mu$ is taken in the form $\mu_{i j k l}=\mu(\mathbf{x}) \delta_{k j} \delta_{i l}$ we can recover a simpler equation, for a scalar medium, that could correspond (in two dimensions) to an antiplane shear wave in a medium of constant density $\rho=1$, and fluctuating shear modulus $\mu$. In this case $\mathbf{G}$ represents antiplane displacement. The simplified version could also correspond to an acoustic pressure wave in a medium with constant bulk modulus, and fluctuating material density $1 / \rho=\mu$. Neither of these interpretations of the similar version of Eq. (1) correspond to a practical system, but such interpretation may nevertheless be of utility for numerical simulations, as a scalar analog to the elastic wave equation for a medium with constant material density and fluctuating moduli, much like a polycrystal.

The perturbative technique to be employed considers effective properties to leading order in the strength of the fluctuations. As their mean $\langle\gamma\rangle$ is zero, the leading order nontrivial quantity is the moduli-covariance $\Lambda$, such that

$$
\begin{aligned}
& \left\langle\gamma_{i j k l}(\mathbf{x})\right\rangle=0, \\
& \left\langle\gamma_{i j k l}(\mathbf{x}) \gamma_{\alpha \beta \gamma \delta}(\mathbf{y})\right\rangle=\Lambda(\mathbf{x}-\mathbf{y})_{i j k l}^{\alpha \beta \gamma \delta},
\end{aligned}
$$

where the angular brackets \langle\rangle are used to define the ensemble average quantities. On the assumption of statistical homogeneity, the moduli covariance has been taken to depend only on the difference between vector $\mathbf{x}$ and $\mathbf{y}$. In previous work we have taken the modulus covariance to factor into a scalar function of distance $|\mathbf{x}-\mathbf{y}|$ and a constant eighth rank tensor. ${ }^{16,23}$

The temporal Fourier transform of $\mathbf{G}$ is defined by

$$
G_{i \alpha}(\mathbf{x}, \mathbf{y}, \omega)=\int_{-\infty}^{\infty} G_{i \alpha}(\mathbf{x}, \mathbf{y}, t) \exp (i \omega t) d t,
$$

in which case Eq. (2) becomes

$$
\begin{aligned}
& {\left[(\omega+i \varepsilon)^{2} \delta_{l i}+C_{k l i j}^{0} \partial_{k} \partial_{j}+\frac{\partial}{\partial x_{k}} \gamma_{k l i j}(\mathbf{x}) \frac{\partial}{\partial x_{j}}\right] G_{i \alpha}(\mathbf{x}, \mathbf{y})} \\
& \quad=\delta^{3}(\mathbf{x}-\mathbf{y}) \delta_{l \alpha},
\end{aligned}
$$

where an $i \varepsilon$ has been included to emphasize the infinitesimal positive imaginary part of $\omega$. Causality (the vanishing of $G$ for negative values of $t$ ) assures that the Fourier transform is analytic in the upper half complex $\omega$ plane. Thus one understands $\omega$ to be complex: $\omega \rightarrow \omega+i \varepsilon$, where $\varepsilon$ is an infinitesi- mal positive quantity. This is a common device, and allows one to guarantee the existence of the Fourier transforms of nonsquare integrable functions, and to resolve the corresponding singularities. ${ }^{16,44}$ Note that in Eq. (5) the $\omega$ dependence of $\mathbf{G}$ has been suppressed, but will be included from time to time for clarity.

The mean solution $\langle\mathbf{G}\rangle$ to Eq. (5) is expressible as the solution of an integral equation:

$$
\begin{aligned}
\left\langle G_{i \alpha}(\mathbf{x}, \mathbf{y})\right\rangle= & G_{i \alpha}^{0}(\mathbf{x}, \mathbf{y})+\iint G_{i \beta}^{0}(\mathbf{x}, \mathbf{z}) m_{\beta j}\left(\mathbf{z}, \mathbf{z}^{\prime}\right) \\
& \times\left\langle G_{j \alpha}\left(\mathbf{z}^{\prime}, \mathbf{y}\right)\right\rangle d^{3} z d^{3} z^{\prime},
\end{aligned}
$$

in terms of the bare Green's function, $\mathbf{G}^{0}\left[\mathbf{G}^{0} \equiv\right.$ the solution to Eq. (5) when $\gamma=0]$ and in terms of the self-energy operator $\mathbf{m}$. Equation (6) is termed a Dyson equation. ${ }^{16,45}$

The first-order smoothing approximation for the selfenergy is given by

$$
m_{\beta j}\left(\mathbf{z}, \mathbf{z}^{\prime}\right)=\left\langle\frac{\partial}{\partial z_{\alpha}} \gamma_{\alpha \beta \gamma \delta}(\mathbf{z}) \frac{\partial}{\partial z_{\delta}} G_{\gamma k}^{0}\left(\mathbf{z}, \mathbf{z}^{\prime}\right) \frac{\partial}{\partial z_{i}^{\prime}} \gamma_{i k j l}\left(\mathbf{z}^{\prime}\right) \frac{\partial}{\partial z_{1}^{\prime}}\right\rangle,
$$

which is exact to this leading order in the modulus fluctuations (under the assumption that $\gamma \ll C^{0}$ ).

These expressions are simpler after employing a spatial Fourier transform. The spatial Fourier transform of an operator like $\mathbf{G}$ or $\mathbf{m}$, with two spatial arguments is defined by

$$
\tilde{f}(\mathbf{q}, \mathbf{p})=\frac{1}{(2 \pi)^{3}} \iint f(\mathbf{x}, \mathbf{y}) \exp [-i \mathbf{q} \cdot \mathbf{x}+i \mathbf{p} \cdot \mathbf{y}] d^{3} x d^{3} y .
$$

Thus, the double spatial Fourier transform of $\mathbf{G}^{0}$ is

$$
\begin{aligned}
\widetilde{G}_{i \alpha}^{0}(\mathbf{p}) \delta^{3}(\mathbf{p}-\mathbf{q})= & \frac{1}{(2 \pi)^{3}} \iint d^{3} x d^{3} y \\
& \times \exp [+i \mathbf{q} \cdot \mathbf{x}-i \mathbf{p} \cdot \mathbf{y}] G_{i \alpha}^{0}(\mathbf{x}, \mathbf{y}) .
\end{aligned}
$$

The delta-function character is attributable to the homogeneity of the bare medium: $\mathbf{G}^{0}(\mathbf{x}, \mathbf{y})=\mathbf{G}^{0}(\mathbf{x}-\mathbf{y})$. The Fourier transform in Eq. (9) has an inverse

$$
\begin{aligned}
G_{i \alpha}^{0}\left(\mathbf{x}, \mathbf{x}^{\prime}\right)= & \frac{1}{(2 \pi)^{3}} \iint d^{3} q d^{3} p \\
& \times \exp \left[i \mathbf{p} \cdot \mathbf{x}-i \mathbf{q} \cdot \mathbf{x}^{\prime}\right] \widetilde{G}_{i \alpha}^{0}(\mathbf{p}) \delta^{3}(\mathbf{p}-\mathbf{q}) .
\end{aligned}
$$

$\mathbf{G}^{0}(\mathbf{p})$ is readily constructed by Fourier transforming Eq. (5) for the case $\gamma=0$ and then solving the resulting Christoffel equations

$$
\left[(\omega+i \varepsilon)^{2}-C_{k l i j}^{0} \mathbf{p}_{k} \mathbf{p}_{j}\right] \widetilde{G}_{i \alpha}^{0}(\mathbf{p})=\delta_{l \alpha},
$$

or

$$
\widetilde{\mathbf{G}}^{0}(\mathbf{p})=\left[\mathbf{I}(\omega+i \varepsilon)^{2}-\mathbf{p} \cdot \mathbf{C}^{0} \cdot \mathbf{p}\right]^{-1} .
$$

Equation (12) may be inverted in closed form under some circumstances. If the Voigt moduli are isotropic, $\mathbf{G}^{0}$ may be decomposed into longitudinal and transverse parts, 


$$
\begin{aligned}
G_{i \alpha}^{0}(\mathbf{p})= & \frac{1}{(\omega+i \varepsilon)^{2}-c_{L}^{2} p^{2}} \hat{p}_{i} \hat{p}_{\alpha}+\frac{1}{(\omega+i \varepsilon)^{2}-c_{T}^{2} p^{2}} \\
& \times\left\{\delta_{i \alpha}-\hat{p}_{i} \hat{p}_{\alpha}\right\} .
\end{aligned}
$$

The statistical homogeneity and isotropy of the medium also implies that the self-energy must have a double spatial Fourier transform with a delta-function form

$$
\begin{aligned}
-\sigma_{\beta j}(p) \delta^{3}(\mathbf{p}-\mathbf{q})= & \frac{1}{(2 \pi)^{3}} \iint d^{3} x d^{3} y \\
& \times \exp \{-i \mathbf{p} \cdot \mathbf{x}+i \mathbf{q} \cdot \mathbf{y}\} m_{\beta j}(\mathbf{x}, \mathbf{y}) .
\end{aligned}
$$

It also implies this form for the mean Green's function

$$
\begin{aligned}
\left\langle\widetilde{G}_{i \alpha}(\mathbf{p})\right\rangle \delta^{3}(\mathbf{p}-\mathbf{q})= & \frac{1}{(2 \pi)^{3}} \iint d^{3} x d^{3} y \\
& \times \exp \{-i \mathbf{p} \cdot \mathbf{x}+i \mathbf{q} \cdot \mathbf{y}\}\left\langle G_{i \alpha}(\mathbf{x}, \mathbf{y})\right\rangle .
\end{aligned}
$$

The Dyson equation is easily solved in the spatial Fourier transform domain,

$$
\begin{aligned}
\left\langle\widetilde{G}_{i \alpha}(\mathbf{p})\right\rangle & =\widetilde{G}_{i \alpha}^{0}(\mathbf{p})-\widetilde{G}_{i \beta}^{0}(\mathbf{p}) \sigma_{\beta k}(\mathbf{p})\left\langle\widetilde{G}_{k \alpha}(\mathbf{p})\right\rangle, \\
\langle\widetilde{\mathbf{G}}(\mathbf{p})\rangle & =\left[\left[\widetilde{\mathbf{G}}^{0}(\mathbf{p})\right]^{-1}+\boldsymbol{\sigma}(\mathbf{p})\right]^{-1} \\
= & {\left[\mathbf{I}(\omega+i \varepsilon)^{2}-\mathbf{p} \cdot \mathbf{C}^{0} \cdot \mathbf{p}+\boldsymbol{\sigma}(\mathbf{p})\right]^{-1} . }
\end{aligned}
$$

The effective wave number $\mathbf{p}$ is the wave number of the mean Green's function, the value of $\mathbf{p}$ at which $\langle\mathbf{G}\rangle$ is singular. These are the solutions $\mathbf{p}$ of the algebraic equation

$$
\operatorname{det}\left[\mathbf{I}(\omega+i \varepsilon)^{2}-\mathbf{p} \cdot \mathbf{C}^{0} \cdot \mathbf{p}+\boldsymbol{\sigma}(\mathbf{p})\right]=0 .
$$

Thus, the problem of determining the mean response reduces to that of determining the self-energy. The real part of $\mathbf{p}$ is inversely proportional to the wave speed and the imaginary part is proportional to the attenuation coefficient $\alpha$.

When the self-energy is given by the first-order smoothing approximation, ${ }^{45}$ the Fourier transform of the self-energy (Eq. (7)) is

$$
\sigma_{\beta j}(\mathbf{p})=p_{\alpha} p_{l} \int G_{\gamma k}^{0}(\mathbf{r}) \frac{\partial}{\partial r_{\delta}} \frac{\partial}{\partial r_{i}}\left[\exp \{-i \mathbf{p} \cdot \mathbf{r}\} \Lambda(\mathbf{r})_{l j k i}^{\alpha \beta \gamma \delta}\right] d^{3} r,
$$

where extensive use has been used made of integration by parts.

By defining the spatial Fourier transform of $\Lambda$ as

$$
\tilde{\Lambda}(\mathbf{p})_{i j k l}^{\alpha \beta \gamma \delta}=\frac{1}{(2 \pi)^{3}} \int \Lambda(\mathbf{r})_{i j k l}^{\alpha \beta \gamma \delta} \exp \{-i \mathbf{p} \cdot \mathbf{r}\} d^{3} r,
$$

we can reexpress $\sigma$ as a convolution in wave number space between $\mathbf{G}^{0}$ and $\Lambda$,

$$
\sigma_{\beta j}(\mathbf{p})=-p_{\alpha} p_{l} \int s_{\delta} s_{i} \widetilde{G}_{\gamma k}^{0}(\mathbf{s}) \widetilde{\Lambda}_{l j k i}^{\alpha \beta \gamma \delta}(\mathbf{p}-\mathbf{s}) d^{3} s .
$$

The Dyson equation governs the evolution of the mean field. Attenuations are then obtained from the imaginary part of the effective wave number. The mean square response, the quantity needed to describe diffuse-field measurements, is proportional to the covariance of the mean Green's function. Therefore, the covariance of the mean Green's functions is now examined using the Bethe-Salpeter equation and ladder approximation.

\section{MATHEMATICAL PRELIMINARIES II: BETHE- SALPETER EQUATION AND THE GREEN'S FUNCTION COVARIANCE}

Calculations of mean responses, related to $\langle\mathbf{G}\rangle$, are not sufficient for the diffuse field measurements in which signals are squared before averaging. For this case, past work has examined the mean square Green's function. In general, however, the quantity of relevance is the Green's function covariance

$$
\left\langle G_{\alpha \beta}\left(\mathbf{x}, \mathbf{x}^{\prime}, \omega\right) G_{i j}^{*}\left(\mathbf{y}, \mathbf{y}^{\prime}, \omega+\Omega\right)\right\rangle,
$$

for which there also exists a multiple scattering formalism. We henceforth suppress the $\omega$ and $\omega+\Omega$ dependencies, taking the asterisk to imply $\omega+\Omega$ as well as complex conjugate. $\left\langle\mathbf{G G}^{*}\right\rangle$ may be inverse spatially Fourier transformed, with respect to all four spatial variables

$$
\begin{aligned}
\underset{\mathbf{p}^{\prime} i}{\mathbf{p} \alpha} \mathbf{H}_{j \mathbf{q}^{\prime}}^{\beta \boldsymbol{q}} \delta^{3}\left(\mathbf{p}+\mathbf{q}^{\prime}-\mathbf{q}-\mathbf{p}^{\prime}\right) \\
=\frac{1}{(2 \pi)^{6}} \int d^{3} x d^{3} x^{\prime} d^{3} y d^{3} y^{\prime}\left\langle G_{\alpha \beta}\left(\mathbf{x}, \mathbf{x}^{\prime}\right) G_{i j}^{*}\left(\mathbf{y}, \mathbf{y}^{\prime}\right)\right\rangle \\
\quad \times \exp \left\{-i \mathbf{p x}+i \mathbf{q} \mathbf{x}^{\prime}+i \mathbf{p}^{\prime} \mathbf{y}-i \mathbf{q}^{\prime} \mathbf{y}^{\prime}\right\},
\end{aligned}
$$

where the delta function is a consequence of the statistical homogeneity of the medium. It is conventional to employ the delta function and a change of variables $\mathbf{p}^{\prime}=\mathbf{p}+\boldsymbol{\Delta}$, and consider only the three wave vector dependent quantity $\underset{\mathbf{q}+\Delta}{\mathbf{q}} \mathbf{H}_{\mathbf{p}+\Delta}^{\mathbf{p}}$.

The covariance is given by the solution of the BetheSalpeter equation ${ }^{16,45}$

$$
\begin{aligned}
& \underset{\mathbf{p}+\Delta i}{\mathbf{p}} H_{j}^{\beta} \underset{\mathbf{q}+\Delta}{\mathbf{q}}={ }_{i}^{\alpha} \boldsymbol{\Gamma}_{j}^{\beta} \underset{\mathbf{q}+\Delta}{\mathbf{q}} \delta^{3}(\mathbf{p}-\mathbf{q}) \\
& +\int d^{3} s_{i}^{\alpha} \Gamma_{k}^{\gamma} \underset{\mathbf{p}+\Delta}{\mathbf{p}} \underset{\mathbf{p}+\Delta k}{\mathbf{p}}{ }_{k}^{\gamma} \mathbf{K}_{l}^{\delta \mathbf{s}+\Delta} \underset{\mathbf{s}+\Delta l}{\mathbf{s}}{ }_{j l}^{\delta} H_{j}^{\beta} \underset{\mathbf{q}+\Delta}{\mathbf{q}},
\end{aligned}
$$

where the double mean field Green's function $\Gamma$ is

$$
{ }_{i}^{\alpha} \Gamma_{k}^{\gamma} \mathbf{q} \mathbf{q}=\left\langle G_{\alpha \gamma}(\mathbf{q})\right\rangle\left\langle G_{i k}^{*}(\mathbf{q}+\mathbf{\Delta})\right\rangle,
$$

which describes the propagation of the square of the mean field. The Bethe-Salpeter equation is formally exact, but approximation is necessary for the operator $\mathbf{K}$ (also known as the intensity operator). In what is often called the ladder approximation because of the shapes of the corresponding Feynman diagrams, $\mathbf{K}$ is approximated, like the mass operator, to leading order in the fluctuating moduli, i.e., to order $\Lambda$,

$$
\underset{\mathbf{p}+\mathbf{\Delta} k}{\mathbf{p}} K_{l}^{\delta \mathbf{s}+\boldsymbol{\Delta}}=p_{\beta} s_{\alpha}\left(p_{i}+\Delta_{i}\right)\left(s_{j}+\Delta_{j}\right) \tilde{\Lambda}(\mathbf{p}-\mathbf{s})_{k i j l}^{\gamma \beta \alpha \delta} .
$$

The Bethe-Salpeter equation may be expanded in a multiple scattering series. To second order in $\mathbf{K}$ it is 


$$
\begin{aligned}
& \underset{\mathbf{p}+\Delta i}{\mathbf{p}} H_{j}^{\beta} \underset{\mathbf{q}+\Delta}{\mathbf{q}+\Delta}={ }_{i}^{\alpha} \Gamma_{j}^{\beta} \underset{\mathbf{q}+\Delta}{\mathbf{q}} \delta^{3}(\mathbf{p}-\mathbf{q}) \\
& +{ }_{i}^{\alpha} \Gamma_{k}^{\gamma} \mathbf{p}+\Delta \underset{\mathbf{p}+\Delta k}{\gamma} K_{l}^{\delta} \underset{\mathbf{q}+\Delta l}{\mathbf{q}} \Gamma_{j}^{\beta}{ }_{j}^{\mathbf{q}+\Delta} \\
& +\int d^{3} s_{i}^{\alpha} \Gamma_{k}^{\gamma \mathbf{p}+\Delta} \underset{\mathbf{p}+\Delta}{\mathbf{p}}{ }_{k}^{\gamma} K_{l}^{\delta \mathbf{s}+\Delta}
\end{aligned}
$$

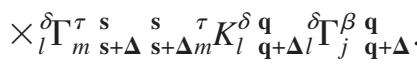

The first term on the right-hand side of Eq. (26) describes a coherent propagation of covariance from source to receiver. The second term describes a coherent propagation at wave vector $\mathbf{q}$, followed by a scattering to wave vector $\mathbf{p}$. The third term in Eq. (26) is composed of five factors. The $\Gamma$ factor on the right describes a coherent propagation, at wave vector $\mathbf{q}$, from the source to the first scattering, the next factor $\mathbf{K}$ describes a scattering to wave vector $\mathbf{s}$, another factor $\Gamma$ describes coherent propagation at wave vector $\mathbf{s}$ to the second scatterer, at which the final wave vector $\mathbf{p}$ is produced. The final factor $\Gamma$, at wave vector $\mathbf{p}$, propagates the covariance coherently to the receiver. These expressions simplify substantially when Feynman diagrams are employed.

The above-mentioned mathematical preliminaries recapitulate, with minor generalizations, some of the material by Weaver. ${ }^{16}$ In that work these equations were applied to the particular case of a point source and a point receiver in a statistically isotropic polycrystal composed of cubic crystallites. A diffusion equation was derived governing the elastic wave energy on sufficiently long time scales, longer than typical times between successive scatterings. An expression for the diffusivity was obtained in terms of microstructural parameters. Later, Turner and Weaver ${ }^{21}$ applied these equations to a less severe limit in which scattering is assumed weak over distances of a wavelength, but in which time scales were not restricted. They transformed the BetheSalpeter equation into a radiative transfer equation similar to Eq. (1). Little attention was paid in that derivation to the roles played by particular sources and receivers, so the ambiguities mentioned in Sec. I were not addressed. Indeed, the purpose there was to derive rationally the otherwise heuristically asserted RTE and thereby to determine expressions for the scattering coefficients in that RTE. In this communication we propose to apply Eqs. (2), (3), and (7) to the particular case of a phase coherent source and receiver, and to determine a radiative-transfer-like equation governing the mean square signal produced at the receiver. This question is the more appropriate one if the intent is to model typical ultrasonic measurements.

\section{SOURCES, RECEIVERS, MEAN, AND MEAN SQUARE SIGNALS}

We consider a medium with a body force that is distributed in space, time, and direction $B_{\alpha}(\mathbf{x}) S(t)$. The factoring into distinct functions of space and time is characteristic of a real transducer, but is not required for subsequent analysis. The source produces a field

$$
\Psi_{\beta}^{S}(\mathbf{x}, t)=\int G_{\beta \alpha}\left(\mathbf{x}, \mathbf{x}^{\prime}, t\right) B_{\alpha}\left(\mathbf{x}^{\prime}\right) \otimes S(t) d^{3} x^{\prime},
$$

written here as a convolution in space and time between the Green's function of the medium and the source, where $\otimes$ represents a temporal convolution. The ensemble average of this response is

$$
\left\langle\Psi_{\beta}^{S}(\mathbf{x}, t)\right\rangle=\int\left\langle G_{\beta \alpha}\left(\mathbf{x}, \mathbf{x}^{\prime}, t\right)\right\rangle B_{\alpha}\left(\mathbf{x}^{\prime}\right) \otimes S(t) d^{3} x^{\prime},
$$

because the source distribution is nonstochastic.

The spatial and temporal Fourier transform of Eq. (28) is

$$
\begin{aligned}
\left\langle\Psi_{\alpha}^{S}(\mathbf{p}, \omega)\right\rangle= & \int \frac{d^{3} x d t}{(2 \pi)^{3 / 2}}\left\langle\Psi_{\alpha}^{S}(\mathbf{x}, t)\right\rangle \exp \{-i \mathbf{p} \cdot \mathbf{x}+i \omega t\} \\
= & \int \frac{d^{3} x d^{3} x^{\prime} d t}{(2 \pi)^{3 / 2}}\left\langle G_{\alpha \beta}\left(\mathbf{x}-\mathbf{x}^{\prime}, t\right)\right\rangle B_{\beta}\left(\mathbf{x}^{\prime}\right) \\
& \otimes S(t) \exp \{-i \mathbf{p} \cdot \mathbf{x}+i \omega t\} \\
= & \int d^{3} r\left\langle G_{\alpha \beta}(\mathbf{r}, t)\right\rangle \exp \{-i \mathbf{p} \cdot \mathbf{r}\} \\
& \times \frac{d^{3} x^{\prime}}{(2 \pi)^{3 / 2}} B_{\beta}\left(\mathbf{x}^{\prime}\right) \exp \{-i \mathbf{p} \cdot \mathbf{x}\} \\
& \otimes S(t) \exp \{i \omega t\} d t \\
= & \left\langle\widetilde{G}_{\alpha \beta}(\mathbf{p}, \omega)\right\rangle \widetilde{B}_{\beta}(\mathbf{p}) S(\omega)
\end{aligned}
$$

Thus the Fourier transformed average field is given by a simple product of the Fourier transform of the source function and the average Green's function.

One can also define a receiver sensitivity distribution function $A_{\alpha}(\mathbf{x}) R(t)$ which when convolved with a field $\Psi$ gives the signal produced by the transducer. If convolved with the Green's function of the medium, it gives the response of the receiver at time $t$ to a point source acting in direction $\beta$ at time zero and position $x$ is

$$
\Psi_{\beta}^{R}(\mathbf{x}, t)=\int R(t) \otimes A_{\alpha}\left(\mathbf{x}^{\prime}\right) G_{\alpha \beta}\left(\mathbf{x}^{\prime}, \mathbf{x}, t\right) d^{3} \mathbf{x}^{\prime} .
$$

Equation (30) can also be averaged and Fourier transformed giving

$$
\begin{aligned}
\left\langle\Psi_{\beta}^{R}(\mathbf{p}, \omega)\right\rangle & =\int \frac{d^{3} x d t}{(2 \pi)^{3 / 2}}\left\langle\Psi_{\beta}^{R}(\mathbf{x}, t)\right\rangle \exp \{i \mathbf{p} \cdot \mathbf{x}+i \omega t\} \\
& =\left\langle\widetilde{G}_{\beta \alpha}(\mathbf{p}, \omega)\right\rangle \tilde{A}_{\alpha}(\mathbf{p}) R(\omega)
\end{aligned}
$$

Note that the spatial Fourier transforms of $\Psi^{S}$ and $\Psi^{R}$ are defined with the opposite sign in the exponent.

If the field produced by the source is detected by the receiver, the resulting signal, $\phi(t)$, is given by the following convolution:

$$
\begin{aligned}
\phi(t)= & \iint R(t) \otimes \mathbf{A}_{\beta}(\mathbf{x}) G_{\beta \alpha}\left(\mathbf{x}, \mathbf{x}^{\prime}, t\right) B_{\alpha}\left(\mathbf{x}^{\prime}\right) \\
& \otimes S(t) d^{3} x d^{3} x^{\prime} .
\end{aligned}
$$

The position of the receiver and source is implicit in the factors $A_{\beta}(\mathbf{x})$ and $B_{\alpha}\left(\mathbf{x}^{\prime}\right)$, which will have their chief support 
in the vicinity of those respective transducers. It is this expression that will serve formally to define the "signal," and of which we will take the mean and mean square. As the source and receiver characteristics are nonstochastic, the mean signal is obtained from Eq. (32) simply by replacing $\mathbf{G}$ with $\langle\mathbf{G}\rangle$. The mean square is more complicated, but clearly entails the covariance.

The temporal Fourier transform of $\langle\phi\rangle$ is

$$
\begin{aligned}
\langle\phi(\omega)\rangle= & \int R(\omega) A_{\alpha}(\mathbf{x})\left\langle G_{\alpha \beta}\left(\mathbf{x}-\mathbf{x}^{\prime}, \omega\right)\right\rangle \\
& \times B_{\beta}\left(\mathbf{x}^{\prime}\right) S(\omega) d^{3} x d^{3} x^{\prime} .
\end{aligned}
$$

On substituting spatial Fourier representations for $A$ and $B$ one obtains

$$
\langle\phi(\omega)\rangle=\int R(\omega) \widetilde{A}_{\alpha}(\mathbf{p})\left\langle G_{\alpha \beta}(\mathbf{p}, \omega)\right\rangle \widetilde{B}_{\beta}(\mathbf{p}) S(\omega) d^{3} p .
$$

Diffuse field techniques typically analyze the square of the signal. Thus we need an expression for $\left\langle\phi^{2}(t)\right\rangle=\Phi(t)$ and its temporal Fourier transform given by

$$
\begin{aligned}
\Phi(\Omega)= & \int_{0}^{\infty} \exp \{-i \Omega t\}\left\langle\phi^{2}(t)\right\rangle d t \\
= & \int_{0}^{\infty} \exp \{-i \Omega t\}\left\langle\phi(t) \phi^{*}(t)\right\rangle d t \\
= & \int_{0}^{\infty} \exp \{-i \Omega t\} \int_{-\infty}^{\infty} \int_{-\infty}^{\infty} \frac{d \omega}{2 \pi} \frac{d \omega^{\prime}}{2 \pi} \\
& \times \exp \{-i \omega t\} \exp \left\{i \omega^{\prime} t\right\}\left\langle\phi(\omega) \phi^{*}\left(\omega^{\prime}\right)\right\rangle d t .
\end{aligned}
$$

After performing the $t$ and $\omega^{\prime}$ integrations, it may be reexpressed in terms of the receiver and source characteristics and Green's function covariance

$$
\begin{aligned}
\Phi(\Omega)= & \int d^{3} x d^{3} x^{\prime} d^{3} y d^{3} y^{\prime} \frac{d \omega}{2 \pi} R(\omega) S(\omega) R^{*}(\omega+\Omega) \\
& \times S^{*}(\omega+\Omega) A_{\alpha}(\mathbf{x}) A_{i}(\mathbf{y}) B_{\beta}\left(\mathbf{x}^{\prime}\right) B_{j}\left(\mathbf{y}^{\prime}\right) \\
& \times\left\langle G_{\alpha \beta}\left(\mathbf{x}, \mathbf{x}^{\prime}\right) G_{i j}^{*}\left(\mathbf{y}, \mathbf{y}^{\prime}\right)\right\rangle,
\end{aligned}
$$

where the covariance of the Green's function can be expressed in terms of its (12-fold) spatial inverse Fourier transform

$$
\begin{aligned}
\left\langle G_{\alpha \beta}\left(\mathbf{x}, \mathbf{x}^{\prime}\right) G_{i j}^{*}\left(\mathbf{y}, \mathbf{y}^{\prime}\right)\right\rangle & \\
= & \frac{1}{(2 \pi)^{6}} \int d^{3} p d^{3} p^{\prime} d^{3} q d^{3} q^{\prime}{ }_{\mathbf{p}^{\prime} i}^{\mathbf{p} \alpha} \mathbf{H}_{j \mathbf{q}^{\prime}}^{\beta \mathbf{q}} \delta^{3}\left(\mathbf{p}+\mathbf{q}^{\prime}-\mathbf{q}-\mathbf{p}^{\prime}\right) \\
& \times \exp \left\{+i \mathbf{p} \cdot \mathbf{x}-i \mathbf{q} \cdot \mathbf{x}^{\prime}-i \mathbf{p}^{\prime} \cdot \mathbf{y}+i \mathbf{q}^{\prime} \cdot \mathbf{y}^{\prime}\right\} .
\end{aligned}
$$

On substituting Eq. (37) into Eq. (36), and performing the integration over space, one obtains

$$
\begin{aligned}
& \Phi(\Omega)=\int \frac{d \omega}{2 \pi} d^{3} p d^{3} q d^{3} \Delta \widetilde{A}_{\alpha}(\mathbf{p}) \widetilde{A}_{i}^{*}(\mathbf{p}+\mathbf{\Delta}) \\
& \quad \times \widetilde{B}_{\beta}(\mathbf{q}) \widetilde{B}_{j}^{*}(\mathbf{q}+\mathbf{\Delta}) \mathbf{p}_{\mathbf{p}+\Delta i}^{\mathbf{p}} H_{j}^{\beta} \mathbf{q} \mathbf{q} \\
& \quad \times R(\omega) R^{*}(\omega+\Omega) S(\omega) S^{*}(\omega+\Omega) .
\end{aligned}
$$

This expression characterizes the mean square signal (in the frequency domain) in terms of the transducer properties and the scattering medium.

\section{SCATTERED RESPONSE}

We now substitute the multiple scattering series of Eq. (26) into Eq. (38) for $\Phi$ and conclude with a multiple scattering series expression for the mean square signal. The zeroth-order term is obtained most easily as

$$
\begin{aligned}
\Phi^{0}(\Omega)= & \int \frac{d \omega}{2 \pi} d^{3} p d^{3} p^{\prime} \tilde{A}_{\alpha}(\mathbf{p}) \tilde{A}_{i}^{*}\left(\mathbf{p}^{\prime}\right) \widetilde{B}_{\beta}(\mathbf{p}) \tilde{B}_{j}^{*}\left(\mathbf{p}^{\prime}\right)_{i}^{\alpha} \Gamma_{j}^{\beta \mathbf{p}} \mathbf{p}^{\prime} \\
& \times R(\omega) R^{*}(\omega+\Omega) S(\omega) S^{*}(\omega+\Omega) \\
= & {\left[\int \frac{d \omega}{2 \pi}\left[d^{3} p \tilde{A}_{\alpha}(\mathbf{p}) \widetilde{B}_{\beta}(\mathbf{p}) R(\omega) S(\omega)\left\langle\widetilde{G}_{\alpha \beta}(\mathbf{p}, \omega)\right\rangle\right]\right.} \\
& \times\left[\int d^{3} p^{\prime} \widetilde{A}_{i}\left(\mathbf{p}^{\prime}\right) \widetilde{B}_{j}\left(\mathbf{p}^{\prime}\right) R(\omega+\Omega) S(\omega+\Omega)\right. \\
& \left.\left.\times\left\langle\widetilde{G}_{i j}\left(\mathbf{p}^{\prime}, \omega+\Omega\right)\right\rangle\right]^{*}\right] \\
= & \int \frac{d \omega}{2 \pi}\langle\phi(\omega)\rangle\langle\phi(\omega+\Omega)\rangle^{*},
\end{aligned}
$$

which implies that

$$
\Phi^{0}(T)=|\langle\phi(T)\rangle|^{2} .
$$

To zeroth order the mean of the square is the square of the mean. A good diffuse field measurement is designed so that the mean response $\langle\phi\rangle$ vanishes. Consequently $\Phi^{0}$ vanishes also.

To first order in powers of $\mathbf{K}, \Phi(\omega)$ is

$$
\begin{aligned}
\Phi^{1}(\Omega)= & \int \frac{d \omega}{2 \pi} d^{3} p d^{3} q d^{3} \Delta\left[\widetilde{A}_{\alpha}(\mathbf{p})\left\langle\widetilde{G}_{\alpha \beta}(p)\right\rangle R(\omega)\right] \\
& \times\left[\widetilde{A}_{i}(\mathbf{p}+\Delta)\left\langle\widetilde{G}_{i j}(\mathbf{p}+\Delta)\right\rangle R(\omega+\Omega)\right]^{*} \\
& \times \underset{\mathbf{p}+\Delta j}{\mathbf{p}} K_{k \mathbf{q}+\Delta}^{\gamma \mathbf{q}}\left[\left\langle\widetilde{G}_{\gamma \tau}(\mathbf{q})\right\rangle \widetilde{B}_{\tau}(p) S(\omega)\right] \\
& \times\left[\left\langle\widetilde{G}_{k l}(\mathbf{q}+\Delta)\right\rangle \widetilde{B}_{l}(p+\Delta) S(\omega+\Omega)\right]^{*} .
\end{aligned}
$$

The quantities in square brackets [] are the Fourier transforms of the (real-valued) fields $\Psi^{R}$ and $\Psi^{S}$. Therefore,

$$
\begin{aligned}
\Phi^{1}(\Omega)= & \int \frac{d \omega}{2 \pi} d^{3} p d^{3} q d^{3} \Delta_{\mathbf{p}+\Delta j}^{\mathbf{p}} K_{k}^{\gamma} \mathbf{q}+\mathbf{q} \\
& \times\left[\int\left\langle\Psi_{\beta}^{R}(\mathbf{x}, t)\right\rangle \frac{d t d^{3} x}{(2 \pi)^{3 / 2}}\right. \\
& \times \exp \{i \mathbf{p} \cdot \mathbf{x}+i \omega t\}]\left[\int\left\langle\Psi_{j}^{R}(\mathbf{y}, u)\right\rangle \frac{d u d^{3} y}{(2 \pi)^{3 / 2}}\right. \\
& \times \exp \{-i(\mathbf{p}+\mathbf{\Delta}) \cdot \mathbf{y}-i(\omega+\Omega) u\}] \\
& \times\left[\int\left\langle\Psi_{\gamma}^{S}\left(\mathbf{x}^{\prime}, t\right)\right\rangle \frac{d t^{\prime} d^{3} x^{\prime}}{(2 \pi)^{3 / 2}} \exp \left\{-i \mathbf{q} \cdot \mathbf{x}^{\prime}+i \omega t^{\prime}\right\}\right]
\end{aligned}
$$

Ghoshal et al:: Diffuse backscatter in heterogeneous media 


$$
\begin{aligned}
& \times\left[\int \langle \Psi _ { k } ^ { S } ( \mathbf { y } ^ { \prime } , u ^ { \prime } ) \rangle \frac { d u ^ { \prime } d ^ { 3 } y ^ { \prime } } { ( 2 \pi ) ^ { 3 / 2 } } \operatorname { e x p } \left\{+i(\mathbf{q}+\mathbf{\Delta}) \cdot \mathbf{y}^{\prime}\right.\right. \\
& \left.\left.-i(\omega+\Omega) u^{\prime}\right\}\right]
\end{aligned}
$$

On making further variable changes

$$
\begin{aligned}
& \mathbf{x}=\mathbf{X}+\boldsymbol{\xi} / 2, \quad \mathbf{y}=\mathbf{X}-\boldsymbol{\xi} / 2, \quad t=T+\tau / 2, \quad u=T-\tau / 2, \\
& \mathbf{x}^{\prime}=\mathbf{X}^{\prime}+\xi^{\prime} / 2, \quad \mathbf{y}^{\prime}=\mathbf{X}^{\prime}-\boldsymbol{\xi}^{\prime} / 2, \quad t^{\prime}=T^{\prime}+\tau^{\prime} / 2, \\
& u^{\prime}=T^{\prime}-\tau^{\prime} / 2,
\end{aligned}
$$

we obtain

$$
\begin{aligned}
\Phi^{1}(\Omega)= & \int \frac{d \omega}{(2 \pi)^{7}} d^{3} p d^{3} q d^{3} \Delta_{\mathbf{p}+\mathbf{\Delta} j}^{\mathbf{p}} K_{k}^{\gamma} \mathbf{\mathbf { q } + \boldsymbol { \Delta }} \\
& \times \int d^{3} X d T d^{3} \xi d \tau\left\langle\psi_{\beta}^{R}(\mathbf{X}+\boldsymbol{\xi} / 2, T+\tau / 2)\right\rangle \\
& \times\left\langle\Psi_{j}^{R}(\mathbf{X}-\boldsymbol{\xi} / 2, T-\tau / 2)\right\rangle \exp \{i \tau(\omega+\Omega / 2) \\
& +i \boldsymbol{\xi} \cdot(\mathbf{p}+\boldsymbol{\Delta} / 2)-i \Omega T-i \mathbf{\Delta} \cdot \mathbf{X}\} \\
& \times \int d^{3} X^{\prime} d T^{\prime} d^{3} \xi^{\prime} d \tau^{\prime}\left\langle\Psi_{\gamma}^{S}\left(\mathbf{X}^{\prime}+\boldsymbol{\xi}^{\prime} / 2, T^{\prime}+\tau^{\prime} / 2\right)\right\rangle \\
& \times\left\langle\Psi_{k}^{S}\left(\mathbf{X}^{\prime}-\boldsymbol{\xi}^{\prime} / 2, T^{\prime}-\tau^{\prime} / 2\right)\right\rangle \exp \left\{i \tau^{\prime}(\omega+\Omega / 2)\right. \\
& \left.-i \boldsymbol{\xi}^{\prime} \cdot(\mathbf{q}+\boldsymbol{\Delta} / 2)-i \Omega T^{\prime}+i \mathbf{\Delta} \cdot \mathbf{X}^{\prime}\right\} .
\end{aligned}
$$

The integrations over $\boldsymbol{\xi}, \tau, \boldsymbol{\xi}^{\prime}$, and $\tau^{\prime}$ can be done by reference to a definition of a four-fold Wigner transform. For source and receiver fields we use opposite sign conventions,

$$
\begin{aligned}
W_{\gamma k}^{S}(\mathbf{X}, T, \mathbf{k}, \omega) & \\
= & \int\left\langle\Psi_{\gamma}^{S}(\mathbf{X}+\xi / 2, T+\tau / 2)\right\rangle \\
& \times\left\langle\Psi_{k}^{S}(\mathbf{X}-\xi / 2, T-\tau / 2)\right\rangle \exp \{-i \mathbf{k} \cdot \boldsymbol{\xi}+i \omega \tau\} d^{3} \xi d \tau, \\
W_{\beta j}^{R}(\mathbf{X}, T, \mathbf{k}, \omega) & \\
= & \int\left\langle\Psi_{\beta}^{R}(\mathbf{X}+\xi / 2, T+\tau / 2)\right\rangle \\
& \times\left\langle\Psi_{j}^{R}(\mathbf{X}-\xi / 2, T-\tau / 2)\right\rangle \exp \{+i \mathbf{k} \cdot \boldsymbol{\xi}+i \omega \tau\} d^{3} \xi d \tau .
\end{aligned}
$$

Equation (45) is spatial and temporal generalizations of the more well known temporal Wigner transform widely discussed in signal processing ${ }^{42,46}$ and others areas such as imaging, ${ }^{47}$ optics,${ }^{48-50}$ and so on. W represents a distribution in space and time $\mathbf{X}, T$ of spectral energy density as a function of wave vector $\mathbf{k}$ and frequency $\omega$.

Using the definitions in Eq. (45) we conclude with an expression for the singly scattered contribution to $\Phi$ :

$$
\begin{aligned}
\Phi^{1}(\Omega)= & \int \frac{d \omega}{(2 \pi)^{7}} d^{3} p d^{3} q d^{3} \Delta_{\mathbf{p}+\Delta j}^{\mathbf{p}} K_{k}^{\gamma} \mathbf{q} \mathbf{q} \mathbf{\Delta} \\
& \times \int d^{3} X d T W_{\beta j}^{R}(\mathbf{X}, T, \mathbf{p}+\mathbf{\Delta} / 2, \omega+\Omega / 2) \\
& \times \exp \{-i \Omega T-i \mathbf{\Delta} \cdot \mathbf{X}\} \\
& \times \int d^{3} X^{\prime} d T^{\prime} W_{\gamma k}^{S}\left(\mathbf{X}^{\prime}, T^{\prime}, \mathbf{q}+\mathbf{\Delta} / 2, \omega+\Omega / 2\right) \\
& \times \exp \left\{-i \Omega T^{\prime}+i \mathbf{\Delta} \cdot \mathbf{X}^{\prime}\right\} .
\end{aligned}
$$

At this point it is necessary to argue that the $\Delta$ and $\Omega$ dependence in $\mathbf{K}$ is negligible. This assumption is the key approximation that allows radiative transfer-like equations to be obtained from the otherwise more general Bethe-Salpeter equation. This then allows the $\Delta$ integration, and the integration over $\Omega$ entailed in the inverse Fourier transform needed to recover $\Phi(t)$, to be done immediately. One finds, after changing variables by means of a shift of $\omega$ by $\Omega / 2$ and a shift of $\mathbf{p}$ and $\mathbf{q}$ by $\Delta / 2$,

$$
\begin{aligned}
\Phi^{1}(T)= & \int \frac{d \omega}{(2 \pi)^{4}} d^{3} p d^{3} q d^{3} X d T^{\prime} \\
& \times W_{\beta j}^{R}\left(\mathbf{X}, T-T^{\prime}, \mathbf{p}, \omega\right){ }_{\mathbf{p} j}^{\mathbf{p} \beta} K_{k}^{\gamma} \mathbf{q}^{\mathbf{q}} W_{\gamma k}^{S}\left(\mathbf{X}, T^{\prime}, \mathbf{q}, \omega\right) .
\end{aligned}
$$

In the ladder approximation for $\mathbf{K}$ as shown in Eq. (25), we see that there is no $\Omega$ dependence. Media for which the inhomgeneities entail factors of $\omega$ (for example, inhomogeneities in mass density) will generate weak $\Omega$ dependence in $\mathbf{K}$; media for which the scatterers have dynamic internal variables (a case in which internal variables are resonant) will generate a stronger $\Omega$ dependence in $\mathbf{K}$, even with the ladder approximation. If one employs a more complex approximation for $\mathbf{K}$ than the ladder approximation, e.g., a $\mathbf{K}$ that includes processes by which there is an internal propagation by $\langle\mathbf{G}\rangle$, there will be an $\Omega$ dependence. All such dependencies correspond to time delays between the arrival of a coherent wave at a scatterer and its subsequent reemision from a scatterer. As we often take the scatterers to have no significant frequency dependence we do not further consider this possibility here.

$\mathbf{K}$ has, even with the ladder approximation, an explicit dependence on $\Delta$. If we were to include the several terms involving $\Delta$, then it would be augmented by extra terms, but with the derivatives of the $\mathbf{W}$ 's with respect to $\mathbf{X}$ in lieu of the W's themselves. These additional terms would be smaller by factors of the ratio of the length scale over which $\mathbf{W}$ varies to the length scale given by $\mathbf{p}$ or $\mathbf{q}$. Thus as long as the Wigner function of the source and receiver fields varies, with $\mathbf{X}$, slowly on the scale of the wavelength, the $\Delta$ dependence in $\mathbf{K}$ is safely neglected. This assumption, that $\Delta \ll \omega / c_{L}$, is the same one that allows the Bethe-Salpeter equation to be recast as an equation of radiative transfer. ${ }^{16}$

This requirement does assert some interesting restraints on the application of Eq. (47). Because W must attenuate like the energy of a coherent field, it implies that the attenuation of the mean Green's function should be moderate or 
small over distances of a wavelength: $\alpha \lambda \ll 2 \pi$. It also implies that the near field of a point source (and the focal region of a sharply focused source) must be a minor part of the multiple scattering process, this is, that very little scattering takes place in any region where the field is changing amplitude over length scales of the order of $\lambda / 2 \pi$. Because the mean free path is equal to or somewhat longer than $1 / 2 \alpha$, this constraint is also equivalent to $\alpha \lambda \ll 2 \pi$.

From Eq. (47), we see that the singly scattered contribution is a convolution in time and space of the product of the Wigner distributions of the beam pattern of the source and receiver, mediated by the scattering strength $\mathbf{K}$ from wave vector $\mathbf{q}$ to wave vector $\mathbf{p}$. The Wigner distributions that enter into this description are the Wigner transforms of the coherent fields associated with source and receiver, the fields that would be present if the source and receiver were placed in a medium described by the average Green's function. Equation (47) is one of the key results of this article.

\section{WIGNER TRANSFORM OF A PISTON TRANSDUCER}

The variance of the signal obtained from a typical $\mathrm{C}$-Scan is defined here as the singly scattered response. In such experiments the signals are acquired at various positions of the transducer and the scattering from the focal depth is analyzed to infer microstructural information by examining the statistics of the signals. The variance of the signal at the focal region may be modeled using Eq. (47). Thompson and Gray previously derived an expression for the singly scattered response (SSR) for polycrystalline materials. ${ }^{51}$ A comparison of their theoretical model with experiment results was first presented by Margetan et al. ${ }^{17}$ Their model was derived for longitudinal-to-longitudinal scattering only. Here, Eq. (47) is used to derive an expression similar to that of Thompson and Gray but within the full multiple-scattering context derived earlier. Such a derivation will allow the limits of the single-scattering assumption to be explored more fully in the future.
Equation (47) is examined for a specific case used extensively for microstructure measurements, for which the same transducer is used as both source and receiver. Since the focus here is on the longitudinal-to-longitudinal SSR, the Wigner transform of the receiver and the source simplify from the generalized tensorial form given by Eq. (45).

The longitudinal component of the mean Green's function in space and time is given by ${ }^{16}$

$$
\left\langle\mathbf{G}\left(\mathbf{x}, \mathbf{x}^{\prime}, t\right)\right\rangle=-\frac{\exp \left[-\alpha^{L}\left|\mathbf{x}-\mathbf{x}^{\prime}\right|\right]}{4 \pi c_{L}^{2}\left(\left|\mathbf{x}-\mathbf{x}^{\prime}\right|\right)} \delta\left(t-\frac{\left|\mathbf{x}-\mathbf{x}^{\prime}\right|}{c_{L}}\right) \hat{\mathbf{p}} \hat{\mathbf{p}}
$$

where $\alpha^{L}$ is the longitudinal attenuation coefficient.

Here, the body force is assumed to be a Gaussian pulse in space at $z=0$ and is given by

$$
B_{\alpha}(\mathbf{x})=B_{0} \delta(z) \exp \left[-\frac{x^{2}+y^{2}}{w_{0}^{2}}\right] \hat{\mathbf{n}}_{\alpha},
$$

where $w_{0}$ is the effective transducer radius, $B_{0}$ is the force per unit area, and $\hat{\mathbf{n}}_{\alpha}$ is the unit normal to the transducer face. Thompson et al. ${ }^{52}$ calculated $w_{0}$ and $B_{0}$ and explained the procedure to obtain the specific values for calibration of a transducer. The source is also assumed to be a Gaussian pulse in time such that

$$
S(t)=\frac{1}{\sigma \sqrt{\pi}} \exp \left[i \omega_{0} t-\frac{t^{2}}{\sigma^{2}}\right],
$$

where $\omega_{0}$ is the forcing frequency and $\sigma$ is the width of the pulse. The mean source field is

$$
\left\langle\Psi_{\beta}(\mathbf{x}, t)\right\rangle=\int\left\langle G_{\beta \alpha}\left(\mathbf{x}, \mathbf{x}^{\prime}, t\right)\right\rangle B_{\alpha}\left(\mathbf{x}^{\prime}\right) \otimes S(t) d^{3} x^{\prime},
$$

where $\otimes$ denotes a temporal convolution. The temporal convolution becomes

$$
\left\langle G_{\beta \alpha}\left(\mathbf{x}, \mathbf{x}^{\prime}, t\right)\right\rangle \otimes S(t)=-\frac{\exp \left(i \omega_{0} t-\left(\frac{t}{\sigma}\right)^{2}\right)}{\sigma \sqrt{\pi} 4 \pi c_{L}^{2}} \frac{\exp \left[\left(-i k_{0}+\frac{2 t}{\sigma^{2} c_{L}}\right)\left|\mathbf{x}-\mathbf{x}^{\prime}\right|-\frac{\left|\mathbf{x}-\mathbf{x}^{\prime}\right|^{2}}{\sigma^{2} c_{L}^{2}}\right]}{\left(\left|\mathbf{x}-\mathbf{x}^{\prime}\right|\right)} \exp \left[-\alpha^{L}\left|\mathbf{x}-\mathbf{x}^{\prime}\right|\right] \hat{p}_{\beta} \hat{p}_{\alpha}
$$

where $\left|\mathbf{x}-\mathbf{x}^{\prime}\right|=\sqrt{\left(z-z^{\prime}\right)^{2}+\left(x-x^{\prime}\right)^{2}+\left(z-z^{\prime}\right)^{2}}$, and $k_{0}=\omega_{0} / c_{L}$. Within the context of the paraxial approximation, the square root is expanded to first order as $\left(\left(z-z^{\prime}\right)+\left\{\left(x-x^{\prime}\right)^{2}+\left(y-y^{\prime}\right)^{2}\right\} / 2 z\right)$ and to zeroth order in the amplitude term as $\left(z-z^{\prime}\right)$. Such an approximation, although not necessary, allows Eq. (47) to be simplified greatly. Thus, under this approximation Eq. (52) becomes

$$
\begin{aligned}
\left\langle G_{\beta \alpha}\left(\mathbf{x}, \mathbf{x}^{\prime}, t\right)\right\rangle \otimes S(t)= & -\frac{1}{\sigma \sqrt{\pi} 4 \pi c_{L}^{2}\left(z-z^{\prime}\right)} \exp \left(i \omega_{0} t-\left(\frac{t}{\sigma}\right)^{2}\right) \exp \left[-\alpha^{L}\left(z-z^{\prime}\right)\right] \\
& \times \exp \left[-i k_{0}\left(\left(z-z^{\prime}\right)+\left\{\left(x-x^{\prime}\right)^{2}+\left(y-y^{\prime}\right)^{2}\right\} / 2 z\right)+\frac{2 t}{\sigma^{2} c_{L}}\left(z-z^{\prime}\right)-\frac{\left(z-z^{\prime}\right)^{2}}{\sigma^{2} c_{L}^{2}}\right] \hat{p}_{\beta} \hat{p}_{\alpha} .
\end{aligned}
$$

Performing the convolution in space reduces Eq. (51) to 


$$
\begin{aligned}
\left\langle\Psi_{\beta}(\mathbf{x}, t)\right\rangle= & -\frac{B_{0} \exp \left(i \omega_{0} t-\left(\frac{t}{\sigma}\right)^{2}\right)}{\sigma \sqrt{\pi} 4 \pi c_{L}^{2}} \exp \left[-\alpha^{L} z+\frac{z\left(2 c_{L} t-z\right)}{\sigma^{2} c_{L}^{2}}\right] \frac{\lambda_{0} w_{0}}{w(z)} \\
& \times \exp \left[-i k_{0} z-\frac{r^{2}}{w^{2}(z)}-i \frac{\pi}{\lambda_{0}} \frac{r^{2}}{R(z)}+i \frac{\pi}{2}+i \Psi_{0}(z)\right] \hat{p}_{\beta}(\hat{\mathbf{p}} \cdot \hat{\mathbf{n}}) .
\end{aligned}
$$

In Eq. (54), $w(z)$ is the Gaussian beam width, $R(z)$ the radius of curvature of the wave front, and $\Psi_{0}(z)$ is the excess phase defined by ${ }^{53}$

$$
\begin{aligned}
& w(z)=w_{0}\left[1+\left(\frac{\lambda_{0} z}{\pi w_{0}^{2}}\right)^{2}\right]^{1 / 2}, \\
& R(z)=z+\left(\frac{\pi w_{0}^{2}}{\lambda_{0}}\right)^{2} \frac{1}{z}, \\
& \Psi_{0}(z)=\tan ^{-1}\left(\frac{\lambda_{0} z}{\pi w_{0}^{2}}\right),
\end{aligned}
$$

where $\lambda_{0}=2 \pi / k_{0}$. The mean source field can also be written in the form

$$
\left\langle\Psi_{\beta}^{S}(\mathbf{r}, z, t)\right\rangle=A(\mathbf{r}, z, t) e^{i \Theta(\mathbf{r}, z, t)} \hat{p}_{\beta}(\hat{\mathbf{p}} \cdot \hat{\mathbf{n}}),
$$

where $r^{2}=x^{2}+y^{2}$. The amplitude $A(\mathbf{r}, z, t)$ and the phase $\Theta(\mathbf{r}, z, t)$ of the source field are given as

$$
\begin{aligned}
A(\mathbf{r}, z, t)= & -\frac{B_{0} \exp \left(-\left(\frac{t}{\sigma}\right)^{2}\right)}{\sigma \sqrt{\pi} 4 \pi c_{L}^{2}} \frac{\lambda_{0} w_{0}}{w(z)} \\
& \times \exp \left[-\frac{r^{2}}{w^{2}(z)}-\alpha^{L} z+\frac{z\left(2 c_{L} t-z\right)}{\sigma^{2} c_{L}^{2}}\right], \\
\Theta(\mathbf{r}, z, t)= & \omega_{0} t-k_{0} z-\frac{\pi}{\lambda_{0}} \frac{r^{2}}{R(z)}+\frac{\pi}{2}+\Psi_{0}(z) .
\end{aligned}
$$

Using high-frequency asymptotics, where it is assumed that $A(\mathbf{r}, z, t)$ varies much slower than $e^{i \Theta(\mathbf{r}, z, t)}$, the Wigner transform of $\left\langle\Psi_{\beta}^{S}\right\rangle$ can be approximated by ${ }^{36,54}$

$$
\begin{aligned}
W(\mathbf{x}, t, \mathbf{k}, \omega)= & (2 \pi)^{4}|A(\mathbf{x}, t)|^{2} \delta^{3}\left(\mathbf{k}-\nabla_{\mathbf{x}} \Theta(\mathbf{x}, t)\right) \\
& \times \delta\left(\omega-\frac{\partial}{\partial t} \Theta(\mathbf{x}, t)\right) .
\end{aligned}
$$

Thus the space-time Wigner transform of the mean field becomes

$$
\begin{aligned}
W_{\gamma k}(\mathbf{x}, \mathbf{k}, t, \omega) \\
=(2 \pi)^{4}\left(\frac{B_{0}}{\sigma \sqrt{\pi} 4 \pi c_{L}^{2}} \frac{\lambda_{0} w_{0}}{w(z)}\right)^{2} \\
\quad \times \exp \left[-2 \alpha^{L} z+2 z \frac{\left(2 c_{L} t-z\right)}{\sigma^{2} c_{L}^{2}}-2 \frac{r^{2}}{w^{2}(z)}-2\left(\frac{t}{\sigma}\right)^{2}\right] \\
\quad \times \delta\left(\omega-\omega_{0}\right) \delta^{2}\left(\mathbf{k}_{r}+\frac{2 \pi}{\lambda_{0}} \frac{r}{R(z)}\right) \delta\left(k_{z}+k_{0}-\frac{\lambda_{0}}{\pi} \frac{1}{w^{2}(z)}\right.
\end{aligned}
$$

$$
\left.-\frac{\pi}{\lambda_{0}} r^{2} \frac{1}{R^{2}(z)}\left(1-\left(\frac{\pi w_{0}^{2}}{\lambda_{0} z}\right)^{2}\right)\right)(\hat{\mathbf{k}} \cdot \hat{\mathbf{n}})^{2} \hat{k} \hat{k}_{k}
$$

The terms in the delta functions

$$
\frac{2 \pi}{\lambda_{0}} \frac{r}{R(z)}, \quad-\frac{\lambda_{0}}{\pi} \frac{1}{w^{2}(z)}-\frac{\pi}{\lambda_{0}} r^{2} \frac{1}{R^{2}(z)}\left(1-\left(\frac{\pi w_{0}^{2}}{\lambda_{0} z}\right)^{2}\right)
$$

are assumed negligible when compared with $k_{0}$. This implies that all the energy is primarily in the $k_{z}=k_{0}$ direction, which is along the transducer axis $\left(|\mathbf{k}|^{2}=k_{r}^{2}+k_{z}^{2} \cong k_{z}^{2}\right)$. Therefore the sidelobes are neglected in what follows. Finally the longitudinal component of the Wigner transform of a piston transducer becomes

$$
\begin{aligned}
W_{\gamma k}^{S}(\mathbf{x}, \mathbf{k}, t, \omega) \\
=(2 \pi)^{4}\left(\frac{B_{0}}{\sigma \sqrt{\pi} 4 \pi c_{L}^{2}} \frac{\lambda_{0} w_{0}}{w(z)}\right)^{2} \\
\quad \times \exp \left[-2 \alpha^{L} z+2 z \frac{\left(2 c_{L} t-z\right)}{\sigma^{2} c_{L}^{2}}-2 \frac{r^{2}}{w^{2}(z)}-2\left(\frac{t}{\sigma}\right)^{2}\right] \\
\quad \times \delta\left(\omega-\omega_{0}\right) \delta^{3}\left(\mathbf{k}+k_{0}\right)(\hat{\mathbf{k}} \cdot \hat{\mathbf{n}})^{2} \hat{k}_{\gamma} \hat{k}_{k} .
\end{aligned}
$$

Equation (61) is the second primary result of this article. It describes the distribution of longitudinal energy in space, time, frequency, and wave vector resulting from a piston transducer. It should be noted that the delta function expressions for frequency and wave vector may correspond to limits of Gaussians, a result that depends on the ansatz for the Wigner transform (e.g., time-space, frequency-space, timewave vector, frequency-wave vector).

\section{SINGLY SCATTERED RESPONSE FROM A PISTON TRANSDUCER}

Using the expression for $\mathbf{W}$ given in Eq. (61) for both the source and receiver, Eq. (47) is now simplified. First the spatial and the tensorial components are assumed to be independent and written as ${ }^{55}$

$$
\tilde{\Lambda}(\mathbf{p})_{l m j k}^{\alpha \beta \gamma \delta}=\Xi_{l m j k}^{\alpha \beta \gamma \delta} \eta(\mathbf{p}),
$$

where $\widetilde{\eta}(\mathbf{p})$ is the Fourier transform of the spatial correlation function. Next the intensity operator is written from the definition in Eq. (25) as (neglecting the slowly varying $\Delta$ dependence)

$$
\begin{aligned}
{ }_{p j}^{p \beta} K_{k q}^{\gamma q} & \approx \eta(\mathbf{p}-\mathbf{q}) \mathbf{p}_{\alpha} \mathbf{q}_{\delta} \mathbf{p}_{l} \mathbf{q}_{m} \Xi_{l m j k}^{\alpha \delta \beta \gamma} \\
& =p^{2} q^{2} \eta(p \hat{\mathbf{p}}-q \hat{\mathbf{q}}) \hat{p}_{\alpha} \hat{q}_{\delta} \hat{p}_{l} \hat{q}_{m} \Xi_{l m j k}^{\alpha \delta \beta \gamma} .
\end{aligned}
$$

The SSR then becomes 


$$
\begin{aligned}
\Phi^{1}(t)= & (2 \pi)^{8}\left(\frac{B_{0} \lambda_{0}}{\sigma \sqrt{\pi} 4 \pi c_{L}^{2}}\right)^{4} \int \frac{d w}{(2 \pi)^{6}}\left[\left(\frac{\omega_{0}}{c_{L}}\right)^{2} \eta\left(\frac{\omega_{0}}{c_{L}} \hat{\mathbf{p}}-\frac{\omega_{0}}{c_{L}} \hat{\mathbf{q}}\right)\right. \\
& \left.\times \hat{p}_{\alpha} \hat{q}_{\delta} \hat{p}_{l} \hat{q}_{m} \Xi_{l m j k}^{\alpha \delta \beta \gamma}\right]\left(\frac{w_{0}}{w(z)}\right)^{4} \\
& \times \exp \left[-4 \alpha^{L} z+2 z \frac{2 c_{L}\left(t-t^{\prime}\right)-z}{\sigma^{2} c_{L}^{2}}+2 z \frac{2 c_{L} t^{\prime}-z}{\sigma^{2} c_{L}^{2}}\right. \\
& \left.-4 \frac{r^{2}}{w^{2}(z)}-2 \frac{\left(t-t^{\prime}\right)^{2}}{\sigma^{2}}-2 \frac{t^{\prime 2}}{\sigma^{2}}\right] \\
& \times \delta^{2}\left(\hat{\mathbf{p}}+\hat{\mathbf{p}}_{0}\right) \hat{p}_{\beta} \hat{p}_{j}(\hat{\mathbf{p}} \cdot \hat{\mathbf{n}})^{2} \delta\left(\omega-\omega_{0}\right) \\
& \times \delta^{2}\left(\hat{\mathbf{q}}+\hat{\mathbf{q}}_{0}\right) \hat{q}_{\gamma} \hat{q}_{k}(\hat{\mathbf{q}} \cdot \hat{\mathbf{n}})^{2} \delta\left(\omega-\omega_{0}\right) d^{2} \hat{p} d^{2} \hat{q} d^{3} X d t^{\prime} .
\end{aligned}
$$

The integration over $\hat{\mathbf{p}}, \hat{\mathbf{q}}$, and $\omega$ are trivial due to the $\delta$ function dependence. In typical diffuse backscatter measurements the angle between the propagation and scattered direction is $\pi$ for a normal incidence pulse echo setup. Therefore, $\hat{\mathbf{p}}_{0} \cdot \hat{\mathbf{n}}=1, \hat{\mathbf{q}}_{0} \cdot \hat{\mathbf{n}}=-1$, and $\hat{\mathbf{p}}_{0} \cdot \hat{\mathbf{q}}_{0}=-1$. Thus the SSR is

$$
\begin{aligned}
\Phi^{1}(t)= & (2 \pi)^{2}\left(\frac{B_{0} \lambda_{0}}{\sigma \sqrt{\pi} 4 \pi c_{L}^{2}}\right)^{4} \int\left(\frac{\omega_{0}}{c_{L}}\right)^{2} \eta\left(\theta_{p_{0} q_{0}}=\pi\right) \\
& \times\left[\hat{p}_{0 \beta} \hat{p}_{0 j} \hat{p}_{0 \alpha} \hat{q}_{0 \delta} \hat{p}_{0 l} \hat{q}_{0 m} \hat{q}_{0 \gamma} \hat{q}_{0 k} \Xi_{l m j k}^{\alpha \delta \beta \gamma}\left(\frac{\omega_{0}}{w(z)}\right)^{4}\right. \\
& \times \exp \left[-4 \alpha^{L} z+4 z \frac{c_{L} t-z}{\sigma^{2} c_{L}^{2}}-4 \frac{r^{2}}{w^{2}(z)}\right. \\
& \left.-2 \frac{\left(t-t^{\prime}\right)^{2}}{\sigma^{2}}-2 \frac{t^{\prime 2}}{\sigma^{2}}\right] d^{3} X d t^{\prime},
\end{aligned}
$$

where $\eta\left(\theta_{p_{0} s_{0}}\right)=\eta\left[\left(\omega / c_{L}\right) \hat{\mathbf{p}}_{0}-\left(\omega / c_{L}\right) \hat{\mathbf{q}}_{0}\right]$ and $\theta_{p_{0} q_{0}}$ is the angle between the vector $\hat{\mathbf{p}}_{0}$ and $\hat{\mathbf{q}}_{0}\left[\hat{\mathbf{p}}_{0} \cdot \hat{\mathbf{q}}_{0}=\cos \left(\theta_{p_{0}} q_{0}\right)\right]$. Let the inner product be denoted in direct form by

$$
\Xi_{l m j k}^{\alpha \delta \beta \gamma} \hat{p}_{\alpha} \hat{p}_{l} \hat{q}_{\delta} \hat{q}_{m} \hat{p}_{\beta} \hat{p}_{j} \hat{q}_{\gamma} \hat{q}_{k}=\Xi_{\ldots . . . \hat{\mathbf{p}} \hat{\mathbf{p}} \hat{\mathbf{p}} \hat{\mathbf{q}} \hat{\mathbf{q}} \hat{\mathbf{q}}}\left(\theta_{p q}\right)
$$

The singly scattered response is then

$$
\begin{aligned}
\Phi^{1}(t)= & (2 \pi)^{2}\left(\frac{B_{0} \lambda_{0}}{\sigma \sqrt{\pi} 4 \pi c_{L}^{2}}\right)^{4}\left[\left(\frac{\omega_{0}}{c_{L}}\right)^{4} \eta(\pi) \Xi \cdots \cdots \hat{p} \hat{\mathbf{p}} \hat{\mathbf{q}} \hat{\mathbf{q}}(\pi)\right] \\
& \times \int\left(\frac{w_{0}}{w(z)}\right)^{4} \exp \left[-4 \alpha^{L} z+4 z \frac{c_{L} t-z}{\sigma^{2} c_{L}^{2}}-4 \frac{r^{2}}{w^{2}(z)}\right. \\
& \left.-2 \frac{\left(t-t^{\prime}\right)^{2}}{\sigma^{2}}-2 \frac{t^{\prime 2}}{\sigma^{2}}\right] d^{3} X d t^{\prime} .
\end{aligned}
$$

The integration in the lateral dimensions, $x$ and $y$, characterizes the effect of scattering from a plane at depth $z$ in the material. ${ }^{17}$ It is given by

$$
\int_{-\infty}^{\infty} \int_{-\infty}^{\infty} \exp \left[-4 \frac{x^{2}+y^{2}}{w^{2}(z)}\right] d x d y=\frac{\pi w^{2}(z)}{4} .
$$

The temporal integration becomes

$$
\int_{-\infty}^{\infty} \exp \left(-2 \frac{\left(t-t^{\prime}\right)^{2}+\left(t^{\prime}\right)^{2}}{\sigma^{2}}\right) d t^{\prime}=\frac{\sigma \sqrt{\pi}}{2} \exp \left[-\frac{t^{2}}{\sigma^{2}}\right] .
$$

Substituting Eqs. (68) and (69) into Eq. (67), the SSR becomes

$$
\begin{aligned}
& \Phi^{1}(t)=\Phi_{0} \frac{1}{w_{0}^{2}}\left[\frac{\pi}{2} \frac{\omega_{0}^{4}}{c_{L}^{8}} \eta(\pi) \Xi_{\ldots . . \hat{\mathbf{p}} \hat{\mathbf{p}} \hat{\mathbf{q}} \hat{\mathbf{q}} \hat{\mathbf{q}} \hat{\mathbf{q}}}(\pi)\right] \exp \left(-\frac{t^{2}}{\sigma^{2}}\right) \\
& \times \int_{-\infty}^{\infty}\left(\frac{\pi}{4} \frac{w_{0}^{4}}{w^{2}(z)}\right) \exp \left(-4 \alpha^{L} z+4 z \frac{c_{L} t-z}{\sigma^{2} c_{L}^{2}}\right) d z,
\end{aligned}
$$

where

$$
\Phi_{0}=\frac{B_{0}^{4} w_{0}^{2}}{4 \sqrt{\pi} \sigma^{3} \omega_{0}^{4}} .
$$

Equation (71) is defined such that $\Phi^{1}(t) / \Phi_{0}$ is a dimensionless quantity. The parameters $\sigma, w_{0}$, and $B_{0}$ in Eq. (71) may be obtained while calibrating and characterizing transducers for typical ultrasonic pulse-echo experiments (i.e., $B_{0}$ can be calculated from the amplitude of the reflected signal from the surface of the test sample). Also, $\sigma$ and $w_{0}$ are related to a Gaussian envelope fit to the input signal.

The integrand in Eq. (70) is related to the transducer beam model. The term in square brackets $[(\pi / 2)$ $\left.\times\left(\omega_{0}^{4} / c_{L}^{8}\right) \eta(\pi) \Xi^{\cdots} \ldots \hat{\mathbf{p}} \hat{\mathbf{p}} \hat{\mathbf{q}} \hat{\mathbf{q}}(\pi)\right]$ is known as the diffuse backscatter coefficient, ${ }^{17,56}$ which depends on the microstructural properties of the material and thus controls the amplitude of the SSR. Equation (70) is the third primary result of this article, a quantity that may be compared directly with experiments involving transducers. In a typical diffuse backscatter experiment the standard deviation of the signals [square root of Eq. (70)] is often used. ${ }^{17}$

In Sec. VIII the SSR model, given by Eq. (70), will be shown to match qualitatively with the Thompson and Gray model. ${ }^{51}$ Their model was developed to include the interface between a liquid and solid boundary, an aspect that is not currently included in the derivation presented here. A rigorous comparison between the two models is now under development. However, it should be noted that differences between the two models are to be expected since the two models are fundamentally different, with the present model based on the fourfold Wigner transform of the transducer beam pattern.

\section{EXAMPLE RESULTS}

Example results are now presented for specific cases of polycrystalline aluminum and iron. The single-crystal material properties used for aluminum are $c_{11}=103.4 \mathrm{GPa}, c_{12}$ $=57.1 \mathrm{GPa}, c_{44}=28.6 \mathrm{GPa}, \rho=2700 \mathrm{~kg} / \mathrm{m}^{3}\left(c_{L}=6289 \mathrm{~m} / \mathrm{s}\right)$ and for iron are $c_{11}=216 \mathrm{GPa}, c_{12}=145 \mathrm{GPa}, c_{44}=129 \mathrm{GPa}$, $\rho=8000 \mathrm{~kg} / \mathrm{m}^{3}\left(c_{L}=5740 \mathrm{~m} / \mathrm{s}\right)$. The ultrasonic beam from a focused transducer converges to a plane known as the focal plane. The best resolution is generally obtained at the focal depth. Hence, the peak amplitude of the scattered response appears near the focal plane in the material. In the following 
examples, the transducer diameter is chosen as $w_{0}$ $=6.35 \mathrm{~mm}$ and the focus into the material is $12 \mathrm{~mm}$.

The SSR given by Eq. (70) for a piston transducer can be easily used for a focused Gaussian beam using the following definitions for the beam width and the curvature: ${ }^{52}$

$$
\begin{aligned}
& q(z)=q(0)+z, \\
& \frac{1}{q(z)}=\frac{1}{R(z)}-\frac{i \lambda_{0}}{\pi w^{2}(z)}, \\
& R(z)=\frac{1}{\operatorname{Re}[1 / q(z)]}, \quad w(z)=\left[\frac{-\lambda_{0} / \pi}{\operatorname{Im}[1 / q(z)]}\right]^{1 / 2},
\end{aligned}
$$

where $R(0)=-F$ with $F$ the focal point. These transformations do not violate any assumptions made previously as long as the focus is not near the transducer surface. Equation (72) reduces to Eq. (55) as $R(0) \rightarrow \infty$ (for a planar transducer the focal point approaches infinity).

The Fourier transform of the two-point correlation function $\eta(r)=e^{-r / L}$ at $\theta_{p q}=\pi$ is given by

$$
\eta(k)=\frac{L^{3}}{\pi^{2}\left(1+k^{2} L^{2}\right)^{2}},
$$

where $L$ is the correlation length. A correlation length of $20 \mu \mathrm{m}$ is used for both materials (for aluminum $\alpha^{L}$ $=0.47 \mathrm{~Np} / \mathrm{m}$; for iron $\alpha^{L}=8.5 \mathrm{~Np} / \mathrm{m}$ at $15 \mathrm{MHz}$ ). Weaver ${ }^{16}$ has given the inner product for scattering from cubic grains which could also be generalized to other material symmetries. The inner product for cubic symmetry is

$$
\exists \ldots . . . \hat{\mathbf{p}} \hat{\mathbf{p}} \hat{\mathbf{q}} \hat{\mathbf{q}} \hat{\mathbf{q}} \hat{\mathbf{q}}\left(\theta_{p q}\right)=\frac{\nu^{2}}{\rho^{2}}\left[\frac{9}{525}+\frac{6}{526} \cos ^{2} \theta_{p q}+\frac{1}{525} \cos ^{4} \theta_{p q}\right],
$$

where $\rho$ is the density and $\nu=c_{11}-c_{12}-2 c_{44}$ for cubic symmetry.

The first example result is that of the Wigner transform for a focused transducer. The spatial and time-dependent part of the Wigner transform in Eq. (61) is given by

$$
\begin{aligned}
A_{1}(r, z, t)= & \frac{1}{w^{2}(z)} \exp \left[-2 \alpha^{L} z+2 z \frac{\left(2 c_{L} t-z\right)}{\sigma^{2} c_{L}^{2}}-2 \frac{r^{2}}{w^{2}(z)}\right. \\
& \left.-2\left(\frac{t}{\sigma}\right)^{2}\right] .
\end{aligned}
$$

Along the transducer axis $(r=0)$, this expression becomes

$$
A_{1}(0, z, t)=\frac{1}{w^{2}(z)} \exp \left[-2 \alpha^{L} z+2 z \frac{\left(2 c_{L} t+z\right)}{\sigma^{2} c_{L}^{2}}-2\left(\frac{t}{\sigma}\right)^{2}\right],
$$

which describes the amplitude of the Wigner transform of the mean source field along the $z$ axis. The contour of this amplitude is shown in Fig. 1 for aluminum. The color bar indicates the amplitude value of the contour. The contour has a peak arrival at the focus $(F=12 \mathrm{~mm})$ at $t=1.91 \mu \mathrm{s}$. The amplitude is maximum around the focal region and approaches zero away from it. The contour is a Gaussian curve along the line defined by $2 c_{L} t-z=0$.

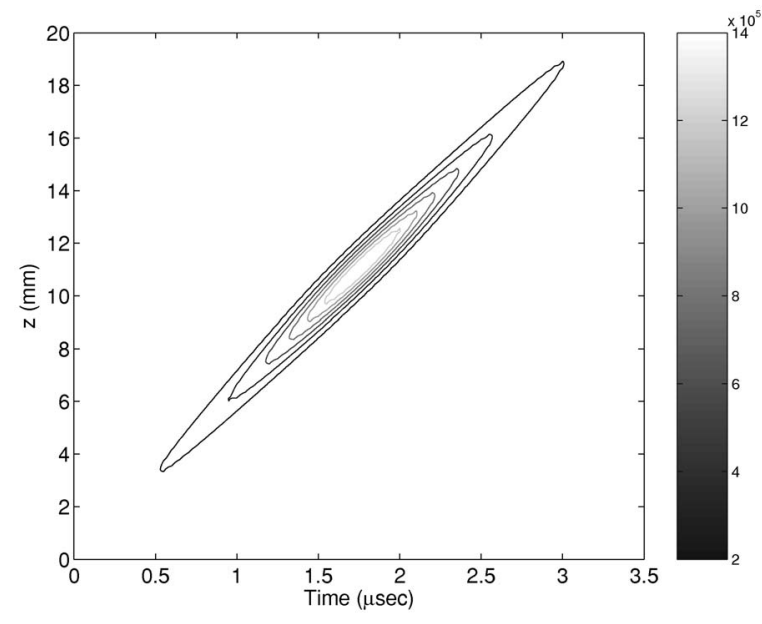

FIG. 1. Contour plot of the $A_{1}(0, z, t)$, defined by Eq. (75) for aluminum.

Example results for the SSR, Eq. (70) normalized by $\Phi_{0}$ are shown in Fig. 2. The central frequency of all the input signals is $15 \mathrm{MHz}$. The SSR given by Eq. (70) depends on the input signal duration $\sigma$. The SSR for various input signals of time duration $t=1,2,3$, and $4 \mu \mathrm{s}$ are shown. From Eq. (70) and the Gaussian beam parameters shown in Eq. (72), the effects of the duration of the input signal on the SSR are shown in Fig. 2. The SSR increases with an increase of the total energy of the input signal. The SSR for $4 \mu \mathrm{s}$ is almost double that of the $1 \mu$ s input signal.

A comparison of the SSR for aluminum and steel is shown in Fig. 3. The amplitude of the singly scattered response increases as the scattering in the material increases. The peak amplitude for steel is higher than for aluminum by more than a factor of 10 . The peak arrival time for the two results also differs since the focal depth depends on the sound speed of the respective material.

Finally, the influence of the focal depth is also examined. The SSR for focal depths of 0.012, 0.02, 0.1, and $1 \mathrm{~m}$ in an aluminum sample are shown in Fig. 4. As the focal length increases and approaches infinity (similar to a planar transducer), the peak in the SSR shifts and finally vanishes. The convergence of the energy at the focal point results in a

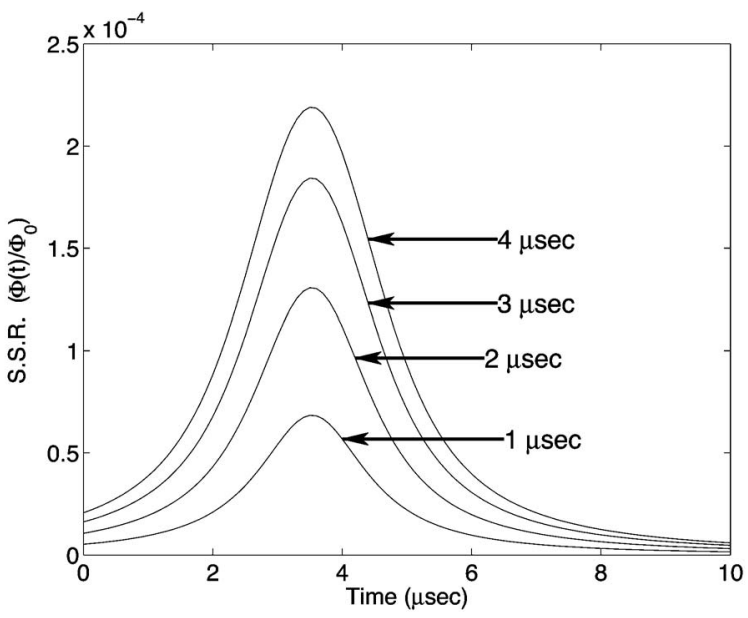

FIG. 2. Normalized singly scattered response (SSR) for input signals of various duration at $15 \mathrm{MHz}$ for aluminum sample. 


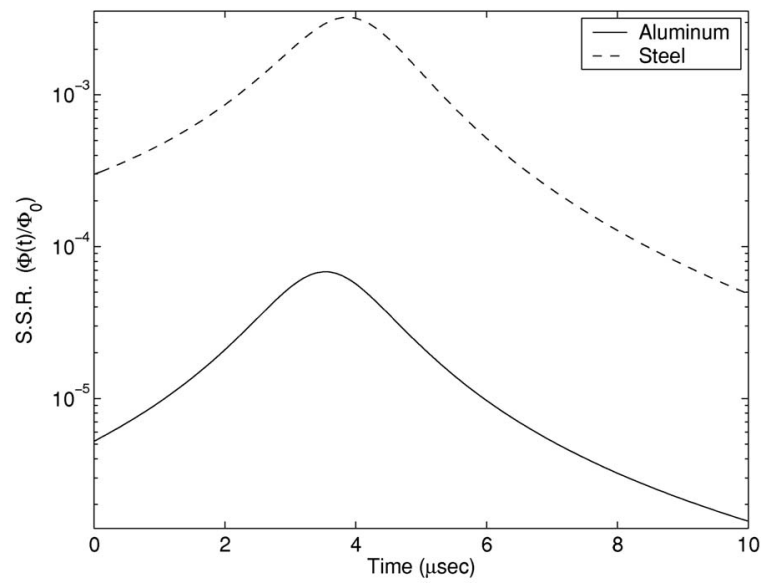

FIG. 3. Normalized SSR in aluminum and steel.

SSR enhancement over that from an unfocused transducer.

\section{SUMMARY}

A theoretical model of SSR in polycrystalline materials for longitudinal-to-longitudinal scattering is presented. The mean response of the medium is deduced using the firstorder smoothing approximation of the self-energy from the Dyson equation. Then the Green's function covariance is derived from the Bethe-Salpeter equation which includes all orders of scattering information, within the context of the ladder approximation. Then the covariance is simplified within a single scattering assumption in conjunction with the Wigner transform of transducer beam model. The Wigner transform of the beam model is proportional to the energy of the source field in the media as a function of space, time, wave vector, and frequency. Finally, the model derived here is tested for a Gaussian beam model with a paraxial approximation and example results are shown for materials of common interest. The model is tested for various parameters (such as the focal depth and duration of the input signal), to observe their influence on the results.

The model for the SSR derived here can be used for microstructural evaluation in the same way that the Thompson and Gray model ${ }^{17}$ has been used: to infer microstructural

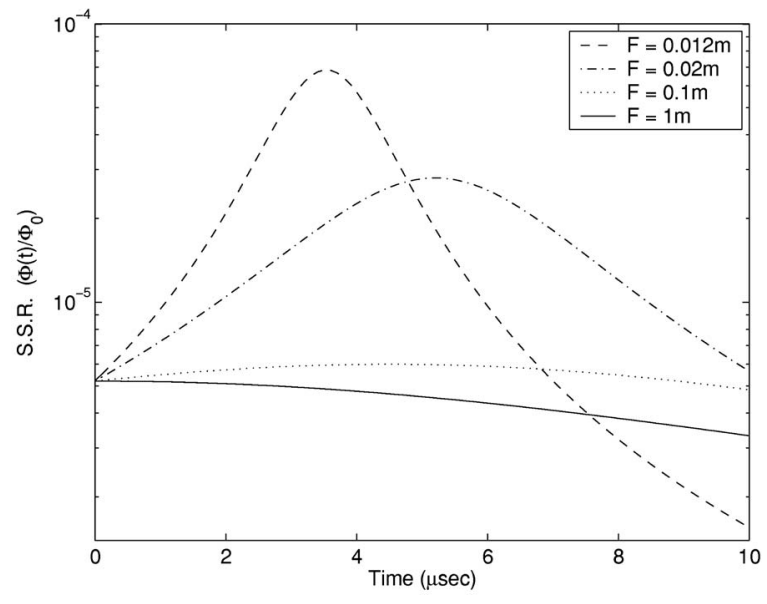

FIG. 4. Normalized SSR for various focal depths in aluminum. information or to identify flaws within a heterogeneous medium. However, one of the impacts of this derivation is associated with the limits of the single-scattering assumption. Since the SSR was derived here within the context of a full multiple scattering formalism, the influence of higher-order scattering can be rigorously examined for realistic measurements (i.e., with a transducer model included in the analysis) as a function of space and time. Thus, experimental data that do not appear to satisfy this assumption may ultimately be understood. Margetan et al. ${ }^{57}$ have shown the effects of multiple scattering in backscatter measurements for a strong scattering material. The present model can also predict the response for a pitch-catch scattering measurement setup where the transmitter and the receiver transducer are oriented at an angle relative to one another. ${ }^{58}$ The pitch-catch method is mainly used to invoke shear wave propagation in the materials. In addition, the development of new, innovative characterization methods that exploit multiple scattering may be anticipated in the future. Finally, it is expected that the derivation of the Wigner distribution for the focused Gaussian beam developed here may be easily generalized to other transducer models, expanding the range of applicability of this derivation. The wide variety of potential new studies of heterogeneous media is exciting indeed.

\section{ACKNOWLEDGMENTS}

The support of the Department of Energy (Grant No. DE-FG02-01ER45890) for this research is gratefully acknowledged. Commercial mathematical software MATLAB ${ }^{59}$ and MATHEMATICA ${ }^{60}$ are used for the numerical calculations presented here.

${ }^{1}$ A. K. Holmes, R. E. Challis, and D. J. Wedlock, "A wide bandwidth study of ultrasound velocity and attenuation in suspensions: Comparison of theory with experimental measurements," J. Colloid Interface Sci. 156, 261-268 (1993).

${ }^{2}$ M. S. Greenwood, J. L. Mai, and M. S. Good, "Attenuation measurements of ultrasound in a Kaolin-Water slurry: A linear dependence on frequency," J. Acoust. Soc. Am. 94, 908-916 (1993).

${ }^{3}$ J. M. Hovem and G. D. Ingram, "Viscous attenuation of sound in saturated sand," J. Acoust. Soc. Am. 66, 1807-1812 (1979).

${ }^{4}$ C. M. Sayers and R. L. Grenfell, "Ultrasonic propagation through hydrating cements," Ultrasonics 31, 147-153 (1993).

${ }^{5} \mathrm{M}$. Biot, "Theory of propagation of elastic waves in a fluid saturated porous solid I," J. Acoust. Soc. Am. 28, 168-178 (1956); 28, 179-191 (1956).

${ }^{6} \mathrm{D}$. Wu, Z. W. Qian, and D. Shao, "Sound attenuation in a coarse granular medium," J. Sound Vib. 162, 529-535 (1993).

${ }^{7}$ R. C. Courtney and L. Mayer, "Acoustic properties of fine grained sediments," J. Acoust. Soc. Am. 93, 3193-3200 (1993).

${ }^{8} \mathrm{~L}$. Schwartz and T. J. Plona, "Ultrasonic propagation in close packed disordered suspensions," J. Appl. Phys. 55, 3971-3977 (1984).

${ }^{9} \mathrm{H}$. Tavossi and F. Cohen-Tenoudji, "Ultrasonic investigation of contact surfaces between grains in random granular media. Effects of variable compression," Proceedings of Ultrasonics International Conference, Vienna, 1993, pp. 347-350.

${ }^{10}$ L. Tsang, J. A. Kong, and T. Habashy, "Multiple scattering of acoustic waves by random distributions of discrete spherical scatterers," J. Acoust. Soc. Am. 71, 552-558 (1982).

${ }^{11}$ J. Liu, L. Ye, D. Weitz, and P. Sheng, "Novel acoustic excitations in suspensions of hard sphere colloids," Phys. Rev. Lett. 65, 2602-2605 (1990).

${ }^{12}$ R. L. Weaver, "Diffuse field decay for materials characterization," in Solid Mechanics Research for Quantitative Non-Destructive Evaluation, edited by J. D. Achenbach and Y. Rajapakse (Martinus Nijhoff, The Hague, 
1987), pp. 425-434.

${ }^{13}$ C. B. Guo, P. Holler, and K. Goebbels, "Scattering of ultrasonic waves in anisotropic polycrystalline metals," Acustica 59, 112-120 (1985).

${ }^{14}$ R. L. Weaver, W. Sachse, K. Green, and Y. Zhang, "Diffuse ultrasound in polycrystalline solids," in Proceedings of Ultrasonics International '91 (Butterworth-Heinemann, Oxford, UK, 1991), pp. 507-510.

${ }^{15}$ R. L. Weaver, "Ultrasonic diffuse field measurements of grain size," in Non-Destructive Testing and Evaluation in Manufacturing and Construction, edited by H. L. M. D. Reis (Hemisphere, New York, 1990), pp. 425-434.

${ }^{16}$ R. L. Weaver, "Diffusivity of ultrasound in polycrystals," J. Mech. Phys Solids 38, 55-86 (1990).

${ }^{17}$ F. J. Margetan, T. A. Gray, and R. B. Thompson, "A technique for quantitative measuring microstructurally induced ultrasonic noise," in Review of Progress in Quantitative NDE, edited by D. O. Thompson and D. E. Chimenti (Plenum, New York, 1991), Vol. 10, pp. 1721-1728.

${ }^{18}$ R. L. Weaver, "Anderson localization of ultrasound," Wave Motion 12 , 129-142 (1990).

${ }^{19}$ R. L. Weaver, "Diffuse waves in finite plates," J. Sound Vib. 94, 319-335 (1984).

${ }^{20}$ R. L. Weaver, "Laboratory studies of diffuse waves in plates," J. Acoust Soc. Am. 79, 919-923 (1986).

${ }^{21}$ J. A. Turner and R. L. Weaver, "Time dependence of multiply scattered diffuse ultrasound in polycrystalline media," J. Acoust. Soc. Am. 97, 2639-2644 (1995).

${ }^{22}$ J. A. Turner and R. L. Weaver, "Radiative transfer of ultrasound," J. Acoust. Soc. Am. 96, 3654-3674 (1994).

${ }^{23}$ J. A. Turner and R. L. Weaver, "Radiative transfer and multiple scattering of diffuse ultrasound in polycrystalline media," J. Acoust. Soc. Am. 96, 3675-3683 (1994)

${ }^{24}$ R. L. Weaver and W. Sachse, "Diffusion of ultrasound in a glass bead slurry," J. Acoust. Soc. Am. 97, 2094-2102 (1995).

${ }^{25}$ A. Tourin, M. Fink, and A. Derode, "Multiple scattering of sound," Waves Random Media 10, R31-R60 (2000).

${ }^{26} \mathrm{P}$. Anugonda, J. S. Wiehn, and J. A. Turner, "Diffusion of ultrasound in concrete," Ultrasonics 39, 429-435 (2001).

${ }^{27}$ A. Ishimaru, Wave Propagation and Scattering in Random Media (Academic, New York, 1978).

${ }^{28}$ L. Tsang, J. A. Kong, and R. T. Shin, Theory of Microwave Remote Sensing (Wiley-Interscience, New York, 1985).

${ }^{29}$ L. Tsang, J. Kong, and K. Ding, Scattering of Electromagnetic Waves: Theory and Applications (Wiley Interscience, New York, 2000).

${ }^{30}$ L. Tsang and J. Kong, Scattering of Electromagnetic Waves: Advanced Topics (Wiley Interscience, New York, 2001).

${ }^{31} 3$ D Radiative Transfer in Cloudy Atmosphere, edited by A. Marshak and A. B. Davis (Springer, Berlin, 2005).

${ }^{32}$ M. I. Mishchenko, L. D. Travis, and A. A. Lacis, Multiple Scattering of Light by Particles: Radiative Transfer and Coherent Backscattering (Cambridge University Press, Cambridge, 2006).

${ }^{33}$ B. Davison, Neutron Transport Theory (Oxford-Clarendon Press, Oxford, 1957).

${ }^{34}$ K. M. Case and P. F. Zweifel, Linear Transport Theory (Addison-Wesley, Reading, 1967).

${ }^{35}$ G. I. Bell and S. Glasstone, Nuclear Transport Theory (Van Nostrand, New York, 1970)

${ }^{36}$ L. Ryzhik, G. Papanicolaou, and J. B. Keller, "Transport equations for elastic and other waves in random media," Wave Motion 24, 327-370 (1996).

${ }^{37}$ N. Trégourès, R. Hennino, C. Lacombe, N. M. Shapiro, L. Margerin, M. Campilo, and B. A. V. Tiggelen, "Multiple scattering of seismic waves," Ultrasonics 40, 269-274 (2002).

${ }^{38}$ A. E. Malcom, J. A. Scales, and B. A. V. Tiggelen, "Extracting the Green function from diffuse, equipartioned waves," Phys. Rev. E 70, 015601 (2004).
${ }^{39}$ N. Trégourès and B. A. V. Tiggelen, "Generalized diffusion equation for multiple scattered elastic waves," Waves Random Media 12, 21-38 (2002).

${ }^{40}$ N. P. Trégourès and B. A. V. Tiggelen, "Quasi-two-dimensional transfer of elastic waves,” Phys. Rev. E 66, 036601 (2002).

${ }^{41}$ S. Chandrasekhar, Radiative Transfer (Dover, New York, 1960).

${ }^{42}$ L. Cohen, Time-Frequency Analysis (Prentice Hall, Englewood Cliffs, NJ, 1995).

${ }^{43}$ E. Wigner, "On the quantum correction for thermodynamic equilibrium," Phys. Rev. 40, 749-759 (1932).

${ }^{44}$ R. L. Weaver, "The effect of an undamped finite degree of freedom 'fuzzy' substructure: Numerical solutions and theoretical discussion," J. Acoust. Soc. Am. 100, 3159-3164 (1996).

${ }^{45}$ U. Frisch, "Wave propagation in random media," in Probabilistic Methods in Applied Mathematics, edited by A. T. Barucha-Reid (Academic, New York, 1968), Vol. 1, pp. 75-198.

${ }^{46}$ P. J. Loughlin and L. Cohen, “A Wigner approximation for wave propagation (L)," J. Acoust. Soc. Am. 118, 1268-1271 (2005).

${ }^{47}$ L. Borcea, G. Papanicolaou, and C. Tsogka, "Adaptive interferometric imaging in clutter and optimal illumination," Inverse Probl. 22, 14051436 (2006).

${ }^{48}$ D. T. Smithey, M. Beck, and M. G. Raymer, "Measurement of the Wigner distribution and the density matrix of a light mode using optical homodyne tomography: Application to squeezed states and the vacuum," Phys. Rev. Lett. 70, 1244-1247 (1993).

${ }^{49}$ A. Wax, S. Bali, and J. E. Thomas, "Time-resolved phase-space distribution for light backscattered from a disordered medium," Phys. Rev. Lett. 85, 66-69 (2000).

${ }^{50}$ F. Reil and J. E. Thomas, "Observation of phase conjugation of light arising from enhanced backscattering in a random media," Phys. Rev. Lett. 95, 143903 (2005).

${ }^{51}$ R. B. Thompson and T. A. Gray, "A model relating ultrasonic scattering measurement through liquid-solid interfaces to unbounded medium scattering amplitudes," J. Acoust. Soc. Am. 74, 1279-1290 (1983).

${ }^{52}$ R. B. Thompson and E. F. Lopes, "The effects of focusing and refraction on Gaussian ultrasonic beams," J. Nondestruct. Eval. 4, 107-123 (1984).

${ }^{53}$ B. D. Cook and W. J. Arnoult III, "Gaussian-Laguerre/Hermite formulation for the nearfield of an ultrasonic transducer," J. Acoust. Soc. Am. 59, 9-11 (1976)

${ }^{54}$ A. D. Kim and A. Ishimaru, "Optical diffusion of focused beam wave pulse in discrete random media," Proc. SPIE 3914, 423-434 (2000).

${ }^{55} \mathrm{~J}$. A. Turner, "Elastic wave propagation and scattering in heterogeneous, anisotropic media: Textured polycrystalline materials," J. Acoust. Soc. Am. 106, 541-552 (1999).

${ }^{56}$ R. B. Thompson, "Elastic wave propagation in random polycrystals: Fundamentals and application to nondestructive evaluation," in Imaging of Complex Media with Acoustic and Seismic Waves, Topics in Applied Physics, edited by M. Fink, W. A. Kuperman, J. Montagner, and A. Tourin (2002), Vol. 84, pp. 233-257.

${ }^{57}$ F. J. Margetan, P. Haldipur, L. Yu, and R. B. Thompson, "Looking for multiple scattering effects in backscattered ultrasonic grain noise from jetengine nickel alloys," Review of Quantitative Nondestructive Evaluation, edited by D. O. Thompson and D. E. Chimenti, (American Institute of Physics, Melville, New York, 2005), Vol. 24, pp. 75-82.

${ }^{58}$ F. J. Margetan, R. Roberts, and R. B. Thompson, "Estimates of signal-tomicrostructural-noise ratios in ultrasonic inspections of metals," Review of Quantitative Nondestrictive Evaluation, edited by D. O. Thompson and D. E. Chimenti, (American Institute of Physics, Melville, NY, 2006), Vol. 25, pp. 1193-1200.

${ }^{59}$ Matlab, The Language of Technical Computing (The Math Works, Inc., Natick, MA, 2001).

${ }^{60}$ Wolfram Research, I., Mathematica, version 4.0 (Wolfram Research, Inc., Champaign, IL, 1999). 